University of Wollongong

Research Online

Faculty of Engineering and Information

Faculty of Engineering and Information

Sciences - Papers: Part A

Sciences

$1-1-2015$

Secondary ionisations in a wall-less ion-counting nanodosimeter:

quantitative analysis and the effect on the comparison of measured and simulated track structure parameters in nanometric volumes

Gerhard Hilgers

Physikalisch-Technische Bundesanstalt (PTB)

Marion Bug

University of Wollongong, mb355@uowmail.edu.au

E Gargioni

UniversitÂ“Atsklinikum Hamburg-Eppendorf

Hans Rabus

Physikalisch-Technische Bundesanstalt (PTB), hans.rabus@ptb.de

Follow this and additional works at: https://ro.uow.edu.au/eispapers

Research Online is the open access institutional repository for the University of Wollongong. For further information contact the UOW Library: research-pubs@uow.edu.au 


\title{
Secondary ionisations in a wall-less ion-counting nanodosimeter: quantitative analysis and the effect on the comparison of measured and simulated track structure parameters in nanometric volumes
}

\author{
Abstract \\ The object of investigation in nanodosimetry is the physical characteristics of the microscopic structure \\ of ionising particle tracks, i.e. the sequence of the interaction types and interaction sites of a primary \\ particle and all its secondaries, which reflects the stochastic nature of the radiation interaction. In view of \\ the upcoming radiation therapy with protons and carbon ions, the ionisation structure of the ion track is of \\ particular interest. Owing to limitations in current detector technology, the only way to determine the \\ ionisation cluster size distribution in a DNA segment is to simulate the particle track structure in \\ condensed matter. This is done using dedicated computer programs based on Monte Carlo procedures \\ simulating the interaction of the primary ions with the target. Hence, there is a need to benchmark these \\ computer codes using suitable experimental data. Ionisation cluster size distributions produced in the \\ nanodosimeter's sensitive volume by monoenergetic protons and alpha particles (with energies between \\ $0.1 \mathrm{MeV}$ and $20 \mathrm{MeV}$ ) were measured at the PTB ion accelerator facilities. C3H8 and N2 were alternately \\ used as the working gas. The measured data were compared with the simulation results obtained with \\ the PTB Monte-Carlo code PTra [B. Grosswendt, Radiat. Environ. Biophys. 41, 103 (2002); M.U. Bug, E. \\ Gargioni, H. Nettelbeck, W.Y. Baek, G. Hilgers, A.B. Rosenfeld, H. Rabus, Phys. Rev. E 88, 043308 (2013)]. \\ Measured and simulated characteristics of the particle track structure are generally in good agreement \\ for protons over the entire energy range investigated. For alpha particles with energies higher than the \\ Bragg peak energy, a good agreement can also be seen, whereas for energies lower than the Bragg peak \\ energy differences of as much as $25 \%$ occur. Significant deviations are only observed for large ionisation \\ cluster sizes. These deviations can be explained by a background consisting of secondary ions. These \\ ions are produced in the region downstream of the extraction aperture by electrons with a kinetic energy \\ of about $2.5 \mathrm{keV}$, which are themselves released by ions of the "primary" ionisation cluster hitting an \\ electrode in the ion transport system. Including this background of secondary ions in the simulated \\ cluster size distributions leads to a significantly better agreement between measured and simulated data, \\ especially for large ionisation clusters. Graphical abstract: [Figure not available: see fulltext.]
}

\section{Keywords}

analysis, quantitative, nanodosimeter, counting, ion, less, wall, ionisations, secondary, volumes, nanometric, parameters, structure, track, simulated, measured, comparison, effect

\section{Publication Details}

Hilgers, G., Bug, M. U., Gargioni, E. \& Rabus, H. (2015). Secondary ionisations in a wall-less ion-counting nanodosimeter: quantitative analysis and the effect on the comparison of measured and simulated track structure parameters in nanometric volumes. European Physical Journal D: Atomic, Molecular, Optical and Plasma Physics, 69 (10), 1-18.

This journal article is available at Research Online: https://ro.uow.edu.au/eispapers/5078 


\title{
Secondary ionisations in a wall-less ion-counting nanodosimeter: quantitative analysis and the effect on the comparison of measured and simulated track structure parameters in nanometric volumes ${ }^{\star}$
}

\author{
Gerhard Hilgers $^{1, a}$, Marion U. Bug${ }^{1,2}$, Elisabetta Gargioni ${ }^{3}$, and Hans Rabus ${ }^{1}$ \\ 1 Physikalisch-Technische Bundesanstalt (PTB), Braunschweig, Germany \\ ${ }^{2}$ Centre for Medical Radiation Physics (CMRP), University of Wollongong, Wollongong, Australia \\ 3 Universitätsklinikum Hamburg-Eppendorf (UKE), Hamburg, Germany
}

Received 16 March 2015 / Received in final form 1st July 2015

Published online 29 October 2015

(C) The Author(s) 2015. This article is published with open access at Springerlink.com

\begin{abstract}
The object of investigation in nanodosimetry is the physical characteristics of the microscopic structure of ionising particle tracks, i.e. the sequence of the interaction types and interaction sites of a primary particle and all its secondaries, which reflects the stochastic nature of the radiation interaction. In view of the upcoming radiation therapy with protons and carbon ions, the ionisation structure of the ion track is of particular interest. Owing to limitations in current detector technology, the only way to determine the ionisation cluster size distribution in a DNA segment is to simulate the particle track structure in condensed matter. This is done using dedicated computer programs based on Monte Carlo procedures simulating the interaction of the primary ions with the target. Hence, there is a need to benchmark these computer codes using suitable experimental data. Ionisation cluster size distributions produced in the nanodosimeter's sensitive volume by monoenergetic protons and alpha particles (with energies between $0.1 \mathrm{MeV}$ and $20 \mathrm{MeV}$ ) were measured at the PTB ion accelerator facilities. $\mathrm{C}_{3} \mathrm{H}_{8}$ and $\mathrm{N}_{2}$ were alternately used as the working gas. The measured data were compared with the simulation results obtained with the PTB Monte-Carlo code PTra [B. Grosswendt, Radiat. Environ. Biophys. 41, 103 (2002); M.U. Bug, E. Gargioni, H. Nettelbeck, W.Y. Baek, G. Hilgers, A.B. Rosenfeld, H. Rabus, Phys. Rev. E 88, 043308 (2013)]. Measured and simulated characteristics of the particle track structure are generally in good agreement for protons over the entire energy range investigated. For alpha particles with energies higher than the Bragg peak energy, a good agreement can also be seen, whereas for energies lower than the Bragg peak energy differences of as much as $25 \%$ occur. Significant deviations are only observed for large ionisation cluster sizes. These deviations can be explained by a background consisting of secondary ions. These ions are produced in the region downstream of the extraction aperture by electrons with a kinetic energy of about $2.5 \mathrm{keV}$, which are themselves released by ions of the "primary" ionisation cluster hitting an electrode in the ion transport system. Including this background of secondary ions in the simulated cluster size distributions leads to a significantly better agreement between measured and simulated data, especially for large ionisation clusters.
\end{abstract}

\section{Introduction}

Radiation-induced damage to tissue is strongly influenced by the pattern of inelastic interactions in sub-cellular targets, especially in the DNA, which is carrying the genetic information. An accumulation of damage, such as that caused by ionisation, in such a short DNA segment leads

\footnotetext{
* Contribution to the Topical Issue "COST Action NanoIBCT: Nano-scale Processes Behind Ion-Beam Cancer Therapy", edited by Andrey Solov'yov, Nigel Mason, Gustavo García, Eugene Surdutovich.

${ }^{a}$ e-mail: gerhard.hilgers@ptb.de
}

to a complex double-strand break (DSB). The larger the number of additional strand breaks within the DNA segment, the more difficult is the repair of the resulting break, and often this repair is error-prone. An irreparable damage to the DNA leads to a programmed cell-death, while in case of misrepair a mutation of the genetic material can occur, which can lead to the generation of radiationinduced cancer $[1,2]$. For an assessment of the biological effectiveness of ionising radiation, the track structure of ionising particles within a target volume of the size of a DNA segment is of particular importance, in particular in view of the upcoming radiation therapy with light ions like protons and carbon ions. 
Owing to limitations in current detector technology, the only way to determine the ionisation cluster size distribution in a DNA segment is to simulate the particle track structure in condensed matter. This is done using dedicated computer programs based on Monte Carlo procedures, which simulate each individual interaction of the primary ions as well as any secondary electrons generated $[3-5]$. In these simulations, the DNA is modelled as a cylindrical target volume comprising water with dimensions corresponding to those of the aforementioned short DNA segment [3,6,7]. Hence, there is a need to benchmark these computer codes using suitable experimental data.

A characteristic parameter for the track structure of an ionising particle is the statistical distribution of the number of ionisations which are produced when a particle traverses a volume element filled with matter. This quantity, referred to as the ionisation cluster size distribution, is amenable to measurement and therefore suitable for benchmarking the aforementioned computer codes. Experimental data for the ionisation cluster size can be obtained with an ion-counting nanodosimeter, which consists basically of a gas-filled counter operated at low pressure.

The measurement procedure for detecting ionisation clusters in diluted gases in coincidence with the original primary particles $[8,9]$ and the development of dedicated Monte Carlo codes for complete particle track structure simulation are the two pillars on which nanodosimetry is based.

\section{Nanodosimetric characteristics of particle track structure}

In nanodosimetry, the ionisation component of the particle track structure is of particular importance, since it is experimentally accessible. It is characterised by the relative frequency distribution of the ionisation cluster size. The ionisation cluster size is defined as the number of ionisations $\nu$ generated in a target volume by a primary particle and its secondary electrons. For reasons of simplicity, this target volume is often chosen to be cylindrical with dimensions comparable to a short segment of DNA as shown in Figure 1.

A primary particle of radiation quality $Q$ can either traverse the target volume or pass it at a distance $d$ with respect to the longitudinal axis of the cylinder. The ionisation cluster size generated in the target can then be obtained using the superposition of the ionisation component of the particle track and of the geometric characteristics of the target volume. The ionisation cluster size distribution is characterised by the statistical distribution of the probabilities $P_{\nu}(Q, d)$ that exactly $v$ ions are created in the target volume. The probability distribution is normalised according to equation (1).

$$
\sum_{\nu=0}^{\infty} P_{\nu}(Q, d)=1 .
$$

For the characterisation of the particle track, however, the statistical moments of the probability distribution are also

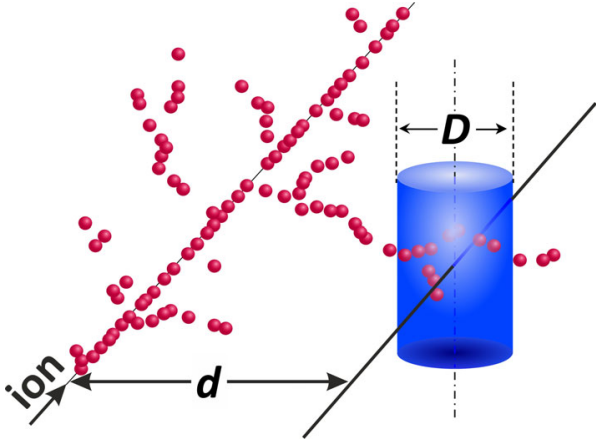

Fig. 1. Schematic representation of the formation of an ionisation cluster by an ionising particle which passes by a cylindrical target volume of diameter $D$ at a distance $d$ from the cylinder axis. In the shown segment of the particle track, the solid circles represent the locations of ionisation interaction.

suited, which are calculated according to equation (2).

$$
M_{\xi}(Q, d)=\sum_{\nu=0}^{\infty} \nu^{\xi} P_{\nu}(Q, d) .
$$

Of particular interest is the first moment of the distribution, that is the mean ionisation cluster size $M_{1}(Q, d)$, which is given by equation (3).

$$
M_{1}(Q, d)=\sum_{\nu=0}^{\infty} \nu P_{\nu}(Q, d) .
$$

The ionisation cluster size distribution $P_{\nu}(Q, d)$ depends on the radiation quality $Q$ (i.e. on the type of the primary particle and its energy) as well as on the geometry of the target volume and its material composition and density.

\section{Setup of the experiment}

The ion-counting nanodosimeter [10] described in Figure 2 comprises an interaction region filled with a rarefied target gas, an electrode system to extract ions from the interaction region, an evacuated acceleration stage with an ion-counting detector at its end, and a primary particle detector.

The actual setup of the experiment allows the measurement of nanodosimetric quantities for primary ion beams, which pass through the sensitive volume perpendicular to its central axis $(d=0)$ as depicted in Figure 1 . In the notation of the quantities $P_{\nu}(Q, d=0)$ and $M_{1}(Q, d=0)$, the index $d=0$ will henceforth be omitted.

The interaction region of the nanodosimeter, which is located between the electrodes of a plane parallel plate capacitor, is filled with the target gas at a pressure in the order of approximately 1 mbar. An ion entering the interaction region through the entrance aperture and traversing it parallel to the two electrodes is registered behind the exit aperture in a semiconductor detector. The positivelycharged ionised gas molecules generated by this primary 


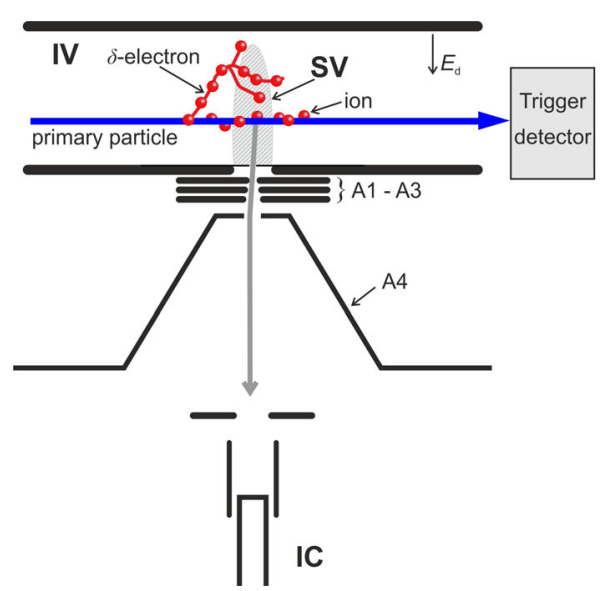

Fig. 2. Schematic representation of the ion-counting nanodosimeter (adapted from [10]). A charged particle passing through the interaction volume (IV) creates ions in the target gas, which are drifted towards the bottom electrode due to the electric field $E_{d}$. Only ions created within the sensitive volume (SV) are extracted via a small aperture in the bottom electrode. These ions are then focused and accelerated via the electrodes A1-A4 into the ion counter (IC), where charge pulses are generated by secondary-electron multiplication. Note that both of the SV and $\delta$-electron track are schematic representations and not to scale.

particle and its secondaries drift towards the lower electrode due to the electrical field, which is applied across the plane parallel plate capacitor. The electrical field strength between the electrodes is selected in such a way that neither the ionised gas molecules nor the electrons originating from the ionisation events generate additional ions. Ions generated in the interaction region's sensitive volume, right above a small aperture in the lower electrode, are extracted from the ionisation region through the lower electrode. They are subsequently transported through an ion optics to an ion-counting secondary electron multiplier, where they are then detected. Prior to detection, the ions are accelerated to a kinetic energy of about $8 \mathrm{keV}$, since the detection probability of the secondary electron multiplier amounts to almost $100 \%$ for ions of this energy originating from the applied target gases [11]. The shape and size of the sensitive volume are defined by the spatial distribution of the efficiency for detecting the generated ions, which is primarily determined by the electrical field strength as well as the target gas and its pressure. Repeated measurement of the generated ions for a large number of single primary particles (of radiation quality $Q$ ), yields the relative frequency distribution $P_{\nu}(Q)$ of the ionisation cluster size $\nu$ of detected ions.

Figure 3 shows the general setup of the PTB nanodosimeter for carrying out measurements at an ion accelerator. To reduce the primary particle rate, the ion beam hits an exchangeable thin gold foil mounted in the scattering chamber at the front of the nanodosimeter. For particle energies below $3 \mathrm{MeV}$, the foil thickness is approximately $100 \mathrm{~nm}$, whereas for higher particle energies, a foil thickness of about $500 \mathrm{~nm}$ is used. Due to Rutherford

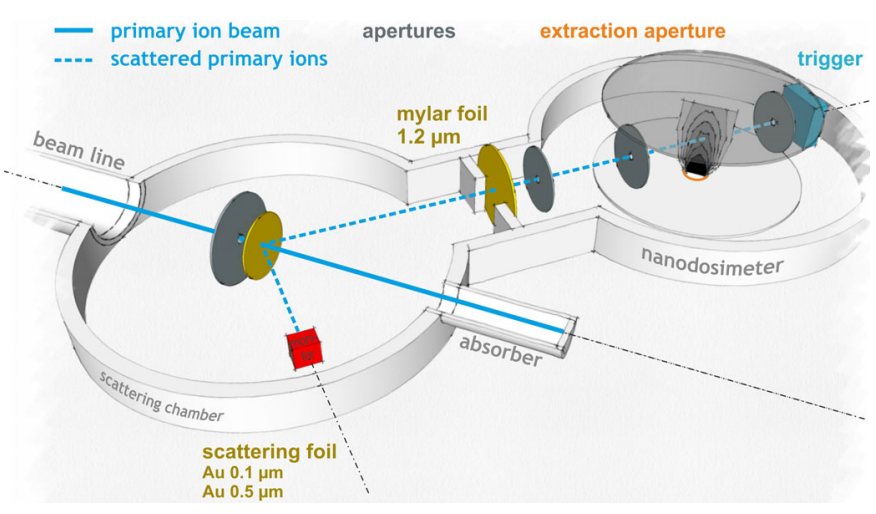

Fig. 3. Schematic setup of the PTB ion-counting nanodosimeter for measurements at an ion accelerator [12].

scattering, a small number of ions is scattered horizontally at an angle of $45^{\circ}$ (relative to the incident beam) towards the entrance aperture of the nanodosimeter or at an angle of $-45^{\circ}$ towards a semiconductor detector which monitors the spectrum of the scattered particles. The unscattered beam is stopped in an absorber, and the primary particles scattered at other angles are absorbed within the walls of the scattering chamber.

A $1.2 \mu$ m-thick Mylar foil (mounted on a $3 \mathrm{~mm}$ diameter aperture) separates the high vacuum of the scattering chamber from the nanodosimeter, thus serving as an entrance window. In order to approximate narrow beam geometry, the diameter of the primary beam is further reduced by a $1 \mathrm{~mm}$ aperture at the entrance of the interaction volume of the nanodosimeter, which is filled with the target gas under investigation.

\section{Data acquisition and evaluation}

Measurements of the ionisation cluster size distributions were carried out with the nanodosimeter operated in pulsed mode [10]. To overcome the counting losses, the data acquisition system described in reference [10] was replaced by a digital storage oscilloscope. This oscilloscope was used to record the waveforms of the collected ions from the ion counter and the charge pulse of the primary particle from the trigger detector. Typical waveforms created by a single primary particle are shown in Figure 4. These waveforms were saved and were then evaluated after the measurements, preserving the correlation between the primary particle and any associated ions that it created.

The pulse height of the charge pulse of the primary particle was determined numerically according to the digital pulse processing algorithm described by Jordanov et al. [13], which allows to use the nanodosimeter's trigger detector as a spectrometer. The waveform of the collected ions from the ion counter was discriminated with a negative voltage threshold to suppress the noise, and the number of ions was determined using a peak detection algorithm.

Using the trigger detector of the nanodosimeter as a spectrometer is advantageous as it allows storage of both 


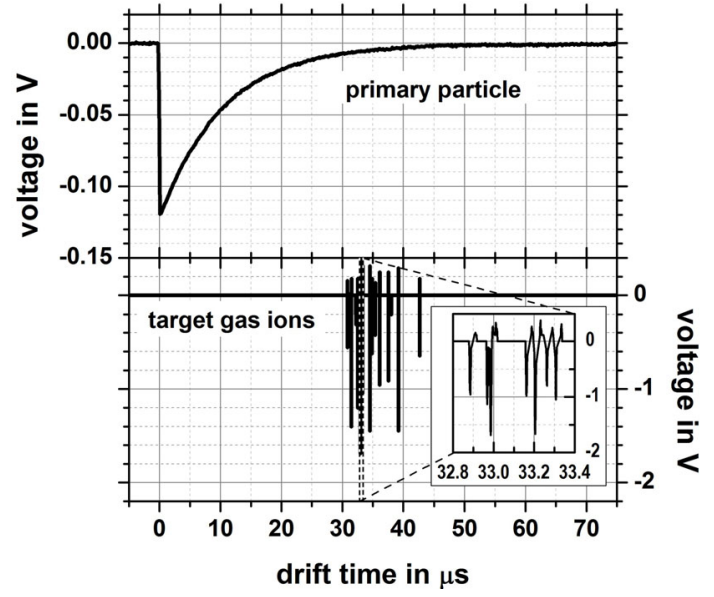

Fig. 4. Measured waveforms of an ionisation cluster of size $\nu=20$ generated by an ${ }^{241} \mathrm{Am}$ alpha particle in $1.2 \mathrm{mbar}$ propane $\left(\mathrm{C}_{3} \mathrm{H}_{8}\right)$ [12]. The upper waveform shows the signal of the charge-sensitive pre-amplifier which is connected to the primary particle detector. The lower waveform shows a sequence of short pulses each of which corresponds to one detected individual ion. As shown in the insert in the lower panel, not all ion pulses can be resolved by eye due to the short time interval between some successive target gas ions.

the energy of the primary particle and the corresponding ionisation cluster size it generated. In the subsequent offline data analysis, a software single channel analyser can then be applied to the energy of the primary particle to discriminate contributions to the cluster size distribution in the case of a mixed field containing particles of different energies.

Recording the waveform of the ion counter instead of only discriminating and counting the pulses allows the creation of a pulse height spectrum for the pulses from the secondary electron multiplier. As any loss of gain due to the ageing of the multiplier leads to a shift of the pulse height spectrum towards lower pulse heights, ageing effects can be compensated by increasing the operating voltage of the multiplier in due time.

To investigate the dependence of the ionisation cluster size distribution on the beam quality, the energy of the primary particles when traversing the sensitive volume must be known. At energies below $0.5 \mathrm{MeV}$ for protons or $2 \mathrm{MeV}$ for alpha particles, the energy loss experienced by the primary particle in the Mylar foil and on its way from the vacuum window to the sensitive volume in the target gas is no longer negligible. By operating the trigger detector as a spectrometer, the energy loss of particles traversing the gas between the vacuum window and the detector can be determined experimentally. By operating the nanodosimeter in the absence of the target gas, an energy calibration of the spectrometer was performed using the pulse height spectra of the primary particles after passing through the gold and Mylar foils. The energy of the primary particles leaving the Mylar foil was calculated with SRIM [14].

For each primary particle, the number of generated ions and their arrival times were recorded relative to

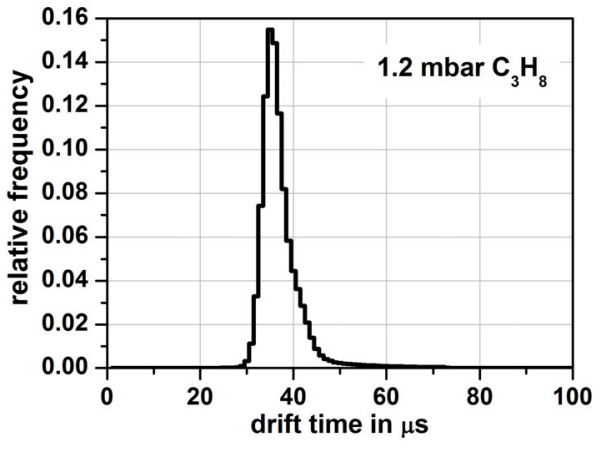

Fig. 5. Measured drift time distribution of the ionisation clusters generated in the nanodosimeter in 1.2 mbar $\mathrm{C}_{3} \mathrm{H}_{8}$ [12].

the detection time of the primary particle. It is important that the ionisation cluster created by a primary particle is fully registered before the next primary particle traverses the ionisation chamber. If a primary particle is detected before the counting of the ions of the previous primary particle has been completed, both events are not taken into account in the evaluation. The acquisition of the ions belonging to a specific primary particle is considered as completed, when the probability of detecting an ion in a specific time interval has dropped to the base line in the drift time distribution. In the distribution shown in Figure 5, the acquisition ends after $70 \mu \mathrm{s}$. The generation of ionisation clusters is measured for a large number $\left(\approx 5 \times 10^{5}\right)$ of primary particles of the same type and energy in order to obtain statistical frequency distributions for the cluster size and the drift time. The drift time distributions show distinct maxima at positions independent of the type and energy of the primary particles. In order to reduce the background, only those ions with drift times lying within a defined time window around their maximum are taken into account in the evaluation.

\section{Extraction efficiency}

Besides the detection probability of the secondary electron multiplier for ions, the measured ionisation cluster size distribution is also influenced by the probability with which the generated ions are extracted from the ionisation chamber, which is dependent on the site of generation. The spatial distribution of this extraction probability defines the sensitive volume. A complex interaction of several factors defines this distribution, namely (i) the form of the electrical flux lines in the vicinity of the extraction aperture, (ii) the transport properties of the ionised gas molecules which are carried along by the electrical field in the (mostly) neutral gas in the interaction region, and (iii) the gas density and flow conditions in the surroundings of the extraction aperture. The spatial distribution of the extraction probability is determined by means of the commercial program package SIMION $^{2} 8.0$,

\footnotetext{
1 http://www.gab.com.au/.

2 http://www.simion. com/.
} 


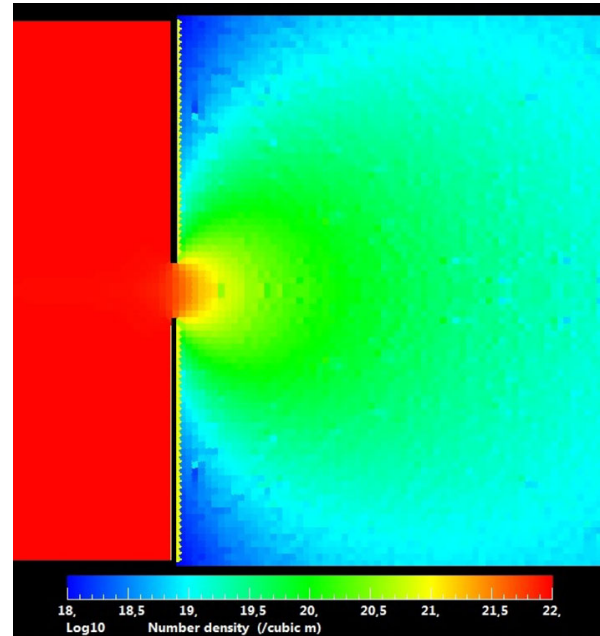

Fig. 6. Screen shot of the gas density in the surroundings of the extraction aperture for the target gas $\mathrm{C}_{3} \mathrm{H}_{8}$ with a pressure of 1.2 mbar in the interaction volume, modelled using the code DS2V $45^{1}$

which was developed for the simulation of electricallycharged particle transport in electrical fields. With additional code, the user can extend SIMION 8.0 to integrate non-implemented transport phenomena. Such an extension was done for the nanodosimeter by including a Monte Carlo simulation of the gas density and flow conditions in the surroundings of the extraction aperture as well as the drift of ionised gas molecules in the neutral gas at a constant pressure. Collisions between the ionised and neutral gas molecules were modelled as elastic hard-sphere collisions. Charge transfer between the ionised and neutral gas molecules was not taken into account, since the simulations did not show significant differences between the absence of charge transfer and maximum charge transfer, i.e. charge transfer occurring in every collision. The gas density (Fig. 6) and flow conditions in the surroundings of the extraction aperture were modelled using the code $\mathrm{DS} 2 \mathrm{~V} 45^{3}$. In the transport simulation of the ionised gas molecules, it was found to be necessary to follow the history of the ionised gas molecule until it reached the entrance aperture of the secondary electron multiplier. Terminating its history when the ionised gas molecule had passed the extraction aperture was insufficient owing to the loss of ionised gas molecules due to scattering with the neutral gas molecules in the effusive jet downstream of the extraction aperture.

The calculated extraction probabilities reveal a significant dependence on the applied electrical field, gas type and gas pressure (see Fig. 7). The position of the primary particle beam is indicated by the dashed white line at a height $h$ of $16.25 \mathrm{~mm}$. For the experimental verification of the spatial distribution of the extraction probability, a spatially resolving detector will be used to detect primary particles, thus allowing the determination of ionisation cluster size distributions as a function of the primary particle's point of impact.

\footnotetext{
${ }^{3}$ http://www.gab.com.au
}
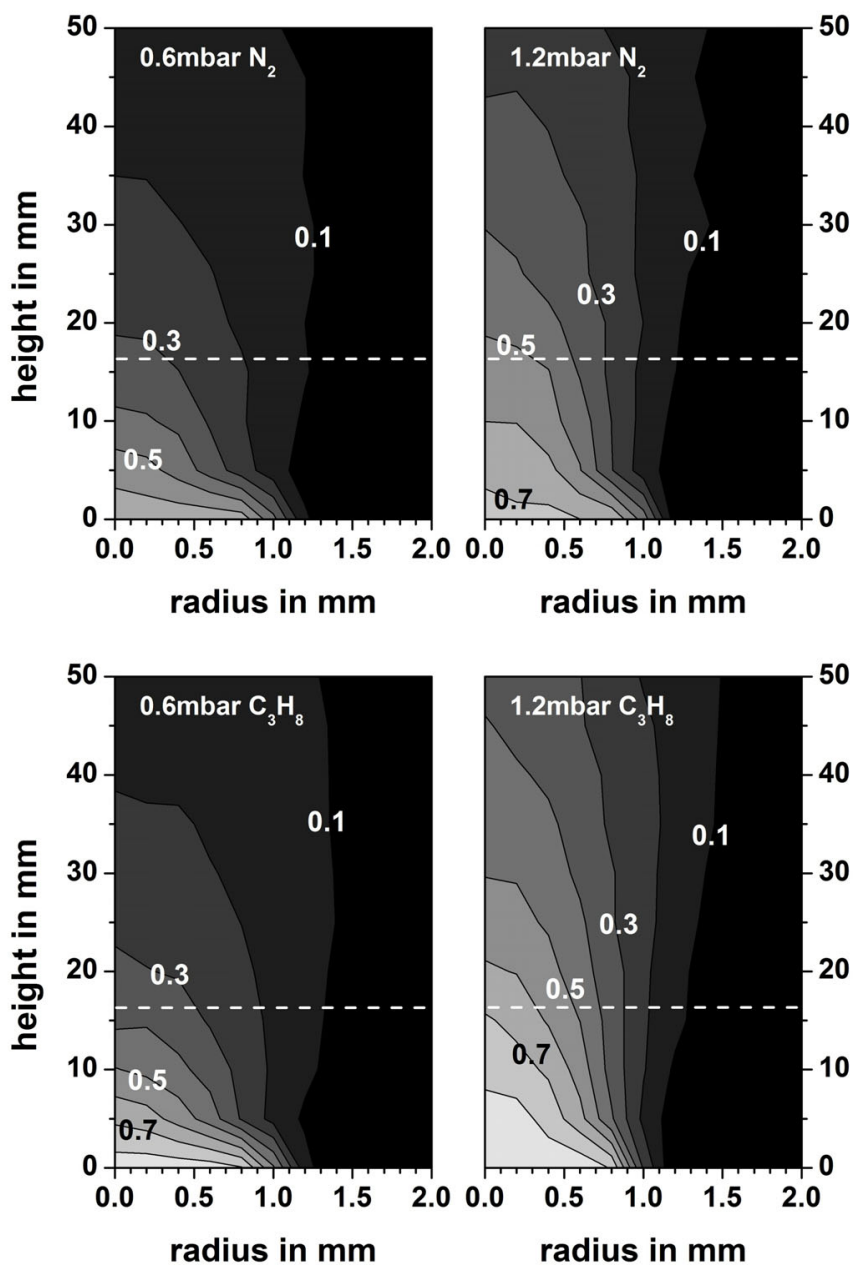

Fig. 7. Calculated extraction probability $\eta$ for nitrogen $\left(\mathrm{N}_{2}\right)$ (top) and propane $\left(\mathrm{C}_{3} \mathrm{H}_{8}\right)$ (bottom) with gas pressures of 0.6 mbar and 1.2 mbar as a function of the radial and vertical distance (i.e. height) from the centre of the extraction aperture [12]. The dashed white line at a height of $16.25 \mathrm{~mm}$ indicates the position of the primary beam. The labels inside the plots denote the values of $\eta$.

Figure 8 shows the mean ionisation cluster size $M_{1}(p)$ produced by alpha particles from an ${ }^{241} \mathrm{Am}$ source for both nitrogen $\left(\mathrm{N}_{2}\right)$ and propane $\left(\mathrm{C}_{3} \mathrm{H}_{8}\right)$ at different target gas pressures $p$ ranging from $0.2 \mathrm{mbar}$ to $1.2 \mathrm{mbar}$. The measured and the simulated data agree within $\pm 9 \%$ for $\mathrm{C}_{3} \mathrm{H}_{8}$, and for $\mathrm{N}_{2}$ the data agree within the experimental uncertainties in the pressure range between 0.8 mbar and 1.2 mbar, whereas for lower pressures the simulation deviates from the measurement by up to $53 \%$ at 0.2 mbar. The overall good agreement indicates the validity of the simulation model for the extraction probability. Additionally, the figure shows the relative extraction probability $\eta$ for the ionised gas molecules integrated along the primary particle trajectory between a radius $r= \pm 2 \mathrm{~mm}$ at a height $h=16.25 \mathrm{~mm}$ multiplied with the target gas pressure $p, p \int \eta(h=16.25 \mathrm{~mm}, p) d r$. This integral contains exclusively the contribution to the mean cluster size of those "onside" ionisations occurring precisely on 

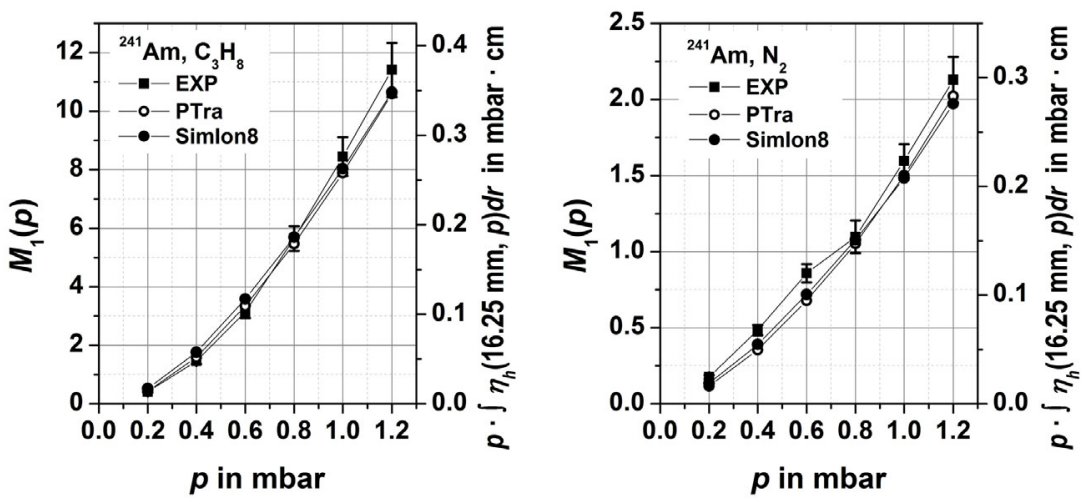

Fig. 8. Measured (EXP) and simulated (PTra) mean ionisation cluster sizes $M_{1}(p)$ and the integrated extraction probability $p \int \eta(h=16.25 \mathrm{~mm}, p) d r$ (SimIon8) of alpha particles from an ${ }^{241} \mathrm{Am}$ source for $\mathrm{C}_{3} \mathrm{H}_{8}$ (left) and $\mathrm{N}_{2}$ (right) at different target gas pressures $p$ ranging from 0.2 mbar to 1.2 mbar.

the primary particle's trajectory, i.e. due to direct interaction of the primary particle with the target, thereby ignoring any surrounding "offside" ionisations, i.e. not along the primary particle's trajectory, such as those due to interactions of secondary particles. However, Monte Carlo simulations with PTra reveal that the difference in the integrated efficiency is less than $8.5 \%$ and $9 \%$ for $\mathrm{N}_{2}$ and $\mathrm{C}_{3} \mathrm{H}_{8}$, respectively, when comparing the integrated efficiency for onside and offside ionisations with respect to the primary particle's trajectory. Therefore, the extraction probability $\eta$ integrated along the primary particle trajectory multiplied with the target gas pressure represents a quantity closely proportional to the mean cluster size $M_{1}(p)$. This can be seen in Figure 8, where the slope of the integrated extraction probability coincides with that of the mean ionisation cluster sizes.

\section{Secondary ionisations}

Transport simulations of the ionised gas molecules revealed a loss of ions due to scattering of the ionised gas molecules with the neutral gas molecules in the effusive jet downstream of the extraction aperture. These scattered ions were found to impinge on the surfaces of the different electrodes downstream of the extraction aperture which are to guide and accelerate the ions towards the secondary electron multiplier. The dominating fraction of the scattered ions was found to hit a cone-shaped electrode (electrode A4 in Fig. 2) with a potential of about $-2.5 \mathrm{kV}$, thus having an energy of about $2.5 \mathrm{keV}$. Ions of this energy create a substantial number of secondary electrons when impinging on a surface. These electrons, created at a potential of $-2.5 \mathrm{kV}$, are accelerated upstream towards the extraction aperture, which is at ground potential. Thus, these electrons gain an energy of up to $2.5 \mathrm{keV}$ in the region immediately downstream of the extraction aperture, where the gas density of the effusive jet is maximal. The accelerated electrons are therefore able to create a significant number of additional ions from the neutral gas molecules forming the effusive gas jet. These additional ions add to the ionised gas molecules originating from ionisation events by the primary particle, thereby increasing the size of the ionisation cluster as contributions from primary ions cannot be distinguished from those of secondary ions. Consequently, the resulting ionisation cluster size comprises a background contribution from secondary ions.

However, since the production of both secondary electrons by ions impinging on a surface and secondary ions by secondary electrons in an extremely inhomogeneous gas target depend on a large number of unknown parameters, a detailed description of the production of secondary ion background is not feasible. Instead, a Poisson distribution was used to describe this background contribution of the secondary ions to the ionisation cluster created by the primary particle. Since the production of secondary ions depends on the effusive gas jet properties and the geometry and potentials of the ion optics downstream of the extraction aperture, the relative contribution of the secondary ion background is independent of ionisation cluster formation and therefore independent of the radiation quality of the primary particle. Thus, for a given target gas and pressure, the secondary ion background can be characterised using two parameters. These are the expectation value $\lambda$ of the Poisson distribution describing the production of the secondary ions, and the probability $\varepsilon$ for an ionised gas molecule from an ionisation cluster to hit the cone shaped electrode after passing through the extraction aperture. Since the secondary ion background is independent of the radiation quality of the primary particle, each of the two parameters $\varepsilon$ and $\lambda$ must have the same value for each cluster size distribution measured with primary particles of any radiation quality for a specific target gas.

\section{Uncertainties}

The uncertainties in the experimental data have effects on the mean ionisation cluster size $M_{1}(Q)$ and the energy determination of primary particles entering the nanodosimeter's sensitive volume. The uncertainties stated in the following are referring to a coverage factor $k=1$ [15]. 


\subsection{Ionisation cluster size}

Measured mean ionisation cluster sizes $M_{1}(Q)$ are determined from an ionisation cluster size distribution, which is the sum of individual cluster size distributions measured for the same target and radiation quality in different measurement shifts. The uncertainties of the total mean ionisation cluster size $M_{1}(Q)$ take into account several contributions: (i) the statistical variation of the mean cluster sizes $M_{1}(Q)$ of the individual cluster size distributions; (ii) the uncertainties of the individual probabilities due to counting statistics; (iii) the uncertainty due to the determination of the target gas density (temperature and pressure measurement) and (iv) an uncertainty contribution related to repeatability determined from constancy measurements carried out repeatedly over a long time period.

The individual contributions to the uncertainty of the mean ionisation cluster size are determined as follows:

Contribution (i): $P_{\nu}(Q)$ is calculated from the measured data as $P_{\nu}(Q)=N_{\nu} / N_{\text {tot }}$, where $N_{\nu}$ is the number of events producing an ionisation cluster of size $\nu$ and $N_{\text {tot }}$ is the total number of recorded events. With $n$ data sets from different measurements using the same radiation quality $Q$ and target, a number of $n$ mean cluster sizes is obtained with $M_{1, k}(Q)$ being the mean cluster size of the $k$ th data set, $k=1, \ldots, n$. With $\bar{M}_{1}(Q)$ being the mean of $n$ values for $M_{1, k}(Q)$, the definition of the mean cluster size (Eq. (3)) yields:

$$
\begin{aligned}
M_{1}(Q) & =\sum_{\nu=0}^{\infty}\left(\nu \frac{N_{\nu}}{N_{t o t}}\right) \\
& =\sum_{\nu=0}^{\infty} \sum_{k=1}^{n} \frac{\nu N_{k, \nu}}{\sum_{\nu=0}^{\infty} \sum_{k=1}^{n} N_{k, \nu}} \\
& =\sum_{k=1}^{n} M_{1, k}(Q) \frac{N_{k}}{N_{t o t}}=\bar{M}_{1}(Q),
\end{aligned}
$$

where

$$
\begin{aligned}
M_{1, k}(Q) & =\sum_{\nu=0}^{\infty}\left(\nu \frac{N_{k, \nu}}{N_{k}}\right) ; N_{k}=\sum_{\nu=0}^{\infty} N_{k, \nu} \\
N_{\nu} & =\sum_{k=1}^{n} N_{k, \nu} ; \quad N_{\text {tot }}=\sum_{\nu=0}^{\infty} \sum_{k=1}^{n} N_{k, \nu}
\end{aligned}
$$

and $N_{k, \nu}$ is the number of events producing an ionisation cluster of size $\nu$ of the $k$ th data set. From equation (4), the amount of contribution (i) to the uncertainty $u\left(M_{1}(Q)\right)$ of $M_{1}(Q)=\bar{M}_{1}(Q)$ due to statistical variations of $M_{1, k}(Q)$ is:

$$
u_{(i)}\left(M_{1}(Q)\right)=\sqrt{\frac{n}{n-1} \sum_{k=1}^{n}\left(M_{1, k}(Q)-M_{1}(Q)\right)^{2} \frac{N_{k}}{N_{\mathrm{tot}}}} .
$$

Contribution (ii): according to the definition of the mean cluster size via the measured number of events (see left hand side of Eq. (4) before the second equals sign), uncertainties of the individual probabilities arise due to counting statistics. This leads to an uncertainty $u_{(i i)}\left(M_{1}(Q)\right)$ given by:

$$
\begin{aligned}
u_{(i i)}\left(M_{1}(Q)\right) & =\sqrt{\sum_{\nu=0}^{\infty}\left(\frac{d\left(\sum_{\nu=0}^{\infty}\left(\nu \frac{N_{\nu}}{N_{\mathrm{tot}}}\right)\right)}{d N_{\nu}} u\left(N_{\nu}\right)\right)^{2}} \\
& =\sqrt{\sum_{\nu=0}^{\infty}\left(\frac{\nu N_{\text {tot }}-\sum_{\nu=0}^{\infty}\left(\nu N_{\nu}\right)}{N_{\mathrm{tot}}^{2}} \sqrt{N_{\nu}}\right)^{2}}
\end{aligned}
$$

using the aforementioned quantities and the uncertainty due to counting statistics:

$$
u\left(N_{\nu}\right)=\sqrt{N}_{\nu}
$$

Contribution (iii): the uncertainty in the determination of the target gas density consists of two components: the uncertainty in the pressure measurement and the uncertainty in the operating temperature of the nanodosimeter. The temperature during the operation of the nanodosimeter was not monitored. However, the experimental hall at the PTB accelerator facilities is temperature stabilised to within $\pm 1{ }^{\circ} \mathrm{C}$. The uncertainty of the pressure measurement is estimated to be $\pm 1 \%$ (see discussion in Sect. 7.3 target gas pressure). Using the pressure dependence of the mean cluster size (Fig. 8), which represents in fact the sensitivity coefficient related to the uncertainty in determining the target gas density, the $1.06 \%$ uncertainty estimate of the gas density transforms into a contribution to the uncertainty of the mean cluster size, which amounts to $1.6 \%$ for 1.2 mbar of $\mathrm{C}_{3} \mathrm{H}_{8}$ and $\mathrm{N}_{2}$, respectively, and to $1.9 \%$ for 0.425 mbar and 0.46 mbar of $\mathrm{C}_{3} \mathrm{H}_{8}$.

Contribution (iv): from constancy measurements carried out repeatedly over a long period of time, an additional $6.9 \%$ uncertainty contribution was taken into account in the determination of the uncertainty of the mean cluster size.

\subsection{Primary particle energy}

The uncertainty in the primary particle energy comprises not only the FWHM of the primary particles' peak in the energy spectrum recorded with the trigger detector, but also the uncertainty due to statistical variation of particle energy and thickness of Mylar and gold foils. The uncertainty due to statistical variation in measuring the particle's energy according to the aforementioned energy calibration procedure for the same target and radiation quality in different measurement shifts was calculated in a similar way to that of $u_{(i)}\left(M_{1}(Q)\right)$ in equation (5). The uncertainty in energy loss is due to the uncertainty in the thickness of the Mylar and gold foils, each of which is estimated to be $10 \%$ of the nominal thickness. The effect of 
this uncertainty on the uncertainty in energy loss was determined via calculation of the corresponding energy loss with SRIM [14]. The amount of this contribution strongly depends on the radiation quality, since the stopping power depends on both the type and energy of the primary ions. Thus, for alpha particles with a primary energy of $1 \mathrm{MeV}$, the uncertainty in the thickness of the foils leads to an energy of $370 \mathrm{keV} \pm 8.9 \%$ when hitting the trigger detector, whereas for $3.5 \mathrm{MeV}$ alpha particles it is $3.12 \mathrm{MeV} \pm 0.6 \%$. In the case of protons, this effect is much less pronounced: for $350 \mathrm{keV}$ protons, the uncertainty in the thickness of the foils leads to an energy of $190 \mathrm{keV} \pm 4.8 \%$ at the trigger detector, whereas for $1 \mathrm{MeV}$ protons, it is $923 \mathrm{keV} \pm 0.43 \%$. The uncertainty in the energy of the primary ions due to the adjustment of the accelerator is less than $0.05 \%$ [16], and hence, it is not taken into account.

\subsection{Target gas pressure}

The pressure in the interaction region is measured by means of a capacitance manometer. The point of measurement is not directly in the sensitive volume but at some distance $(\sim 30 \mathrm{~cm})$ away. The mean free path between two collisions, however, is in the order of a few tens of micrometers for both $\mathrm{N}_{2}$ and $\mathrm{C}_{3} \mathrm{H}_{8}$ in the pressure range under consideration, and thus, the conditions of laminar flow are fulfilled. Considering the trajectory of the incident primary particle is located $16.25 \mathrm{~mm}$ above the extraction aperture, and simulations of the gas density (Fig. 6) and flow conditions in the vicinity of the extraction aperture showed no substantial changes upstream, no significant differences are to be expected between the pressure in the sensitive volume and in the point of pressure measurement.

\subsection{Extraction probability}

The simulated spatial distribution of the extraction probability is an input parameter into the Monte Carlo simulation of the ionisation clusters. For the comparison of measured and simulated cluster size distributions in the present work, it does not affect the data evaluation of the measured ionisation clusters, only the data evaluation of simulated cluster size distributions is affected. It is difficult to estimate the uncertainty associated with the spatial distribution of the extraction probability due to the unknown uncertainties of the transport model implemented in the user code for SIMION 8.0. Nevertheless, it is possible to investigate the effect of varying input parameters for the simulation of this spatial distribution of the extraction probability. A variation of $\pm 5 \%$ in the density of the target gas leads to a change in the product of pressure and efficiency integrated along the primary particle trajectory of $\pm 6.7 \%$ and $\pm 6.8 \%$ for 1.2 mbar of $\mathrm{C}_{3} \mathrm{H}_{8}$ and $\mathrm{N}_{2}$, respectively, and $\pm 8.1 \%$ for $0.425 \mathrm{mbar}$ and 0.46 mbar of $\mathrm{C}_{3} \mathrm{H}_{8}$. Another input parameter for the simulation is the cross section for the collision between the ionised and neutral gas molecules. This parameter is chosen in such a way that the drift time of the ionised gas molecules in the simulation matches the drift time measured in the experiment. A variation in the collision cross section of $\pm 5 \%$ leads to a change in the product of pressure and efficiency integrated along the primary particle trajectory of $\pm 1.0 \%$ and $\pm 1.1 \%$ for 1.2 mbar of $\mathrm{C}_{3} \mathrm{H}_{8}$ and $\mathrm{N}_{2}$, respectively, and of $\pm 3.0 \%$ for $0.425 \mathrm{mbar}$ and 0.46 mbar of $\mathrm{C}_{3} \mathrm{H}_{8}$. The statistical uncertainty in the respective calculations was $0.27 \%$. Nevertheless, the measured and simulated mean cluster sizes $M_{1}(p)$ in $\mathrm{N}_{2}$ and $\mathrm{C}_{3} \mathrm{H}_{8}$ for different target gas pressures (Fig. 8) agree mostly within the experimental uncertainties. For $\mathrm{C}_{3} \mathrm{H}_{8}$, deviations were less than $\pm 9 \%$. For $\mathrm{N}_{2}$, on the other hand, the data agree within the experimental uncertainties in the pressure range between 0.8 mbar and $1.2 \mathrm{mbar}$, but for lower pressures, the simulation deviates from the measurement by as much as $53 \%$ at 0.2 mbar. The overall good agreement is an indication of the quality of the spatial distribution of the extraction probability and the underlying simulation model. Nevertheless, investigations are in progress to improve the description of the spatial distribution of the extraction probability.

\section{Basic check}

The correct operation of the nanodosimeter needs to be verified using a check procedure that is independent of simulation and which can be traced back to fundamentals. A suitable check procedure is based on the slowing down of charged particles in matter. The inelastic cross sections of a substance depend on the type, charge state and energy of the ionising particle being stopped. For particle velocities that are large compared with the velocities of electrons bound in atoms, the inelastic cross sections of ions are identical for different ions of the same charge and velocity according to Bethe's theory [17]. The first moment of the frequency distribution of the number of ionisations, that is the mean ionisation cluster size $M_{1}(Q)$ (given by Eq. (1)), is proportional to the cross section for ionisation by ions. The extraction efficiency $\eta$ is only dependent on the transport properties of the target gas ions in the neutral target gas, and thus, does not depend on how the target gas ions are produced. $M_{1}(Q)$ should therefore be identical for primary particles which are isotopes (i.e. identical atomic number $Z$, but different mass number $A$ ) if the charge states and particle velocities are the same.

Figure 9 shows the comparison of the mean cluster sizes $M_{1}(v)$ as a function of the primary particle velocity $v$ for protons and deuterons in 1.2 mbar $\mathrm{N}_{2}$ and $1.2 \mathrm{mbar}$ $\mathrm{C}_{3} \mathrm{H}_{8}$. For identical velocity $v$, the mean ionisation cluster size $M_{1}(v)$ for protons and deuterons agree within the experimental uncertainties for both target gases, thus verifying the correct basic operation of the nanodosimeter.

\section{Scaling procedure}

Nanodosimetric quantities cannot bemeasured directly in a biological target. This is also true for water, which is 

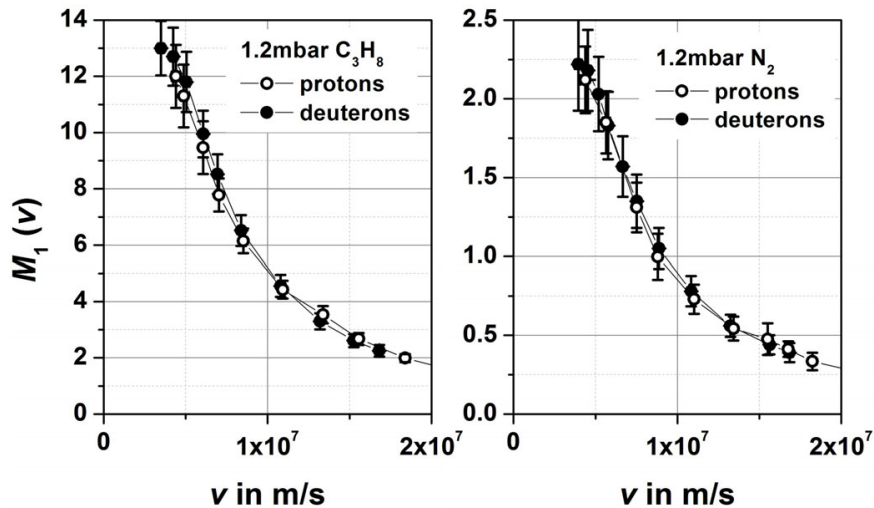

Fig. 9. Comparison of the mean cluster sizes $M_{1}(v)$ as a function of the primary particle velocity $v$ for protons and deuterons in 1.2 mbar $_{3} \mathrm{H}_{8}$ (left) and 1.2 mbar $\mathrm{N}_{2}$ (right).

commonly used as a "biological" reference material for defining nanodosimetric quantities related to radiation quality [3,7]. Experimentally, ionisation cluster size distributions can be assessed using a nanodosimeter, which basically comprises a gas filled counter operating at low pressure. The issue of relating ionisation cluster size distributions of different materials has been addressed by Grosswendt [18], who proposed a simple scaling procedure to relate ionisation cluster size distributions measured in a macroscopic gas target with those calculated by Monte Carlo simulations for nanometric water targets. With this scaling procedure, the gas pressure in the nanodosimeter is chosen such that

$$
(D \rho)^{(\text {gas })}=(D \rho)^{(\text {water })} \frac{(\lambda \rho)_{\text {ion }}^{(\text {gas })}(Q)}{(\lambda \rho)_{\text {ion }}^{(\text {water })}(Q)},
$$

where $D \rho$ is the mass per area, $\lambda_{\text {ion }}$ is the ionisation mean free path of radiation with quality $Q$, and $\rho$ is the material density. In order to have uniquely defined dosimetric quantities, equation (7) should be valid irrespective of radiation quality and selected materials. Thus, an important step towards the direct measurement of cluster size distributions expected in a DNA segment is to test experimentally the scaling procedure defined by equation (7). The validation of equation (7) can be done by comparing measurements with the nanodosimeter for at least two different target gases, which has already been investigated [19]. The aforementioned measured data were used to benchmark the PTra Monte Carlo code (see Fig. 12).

Ionisation cluster size distributions were measured with the nanodosimeter for mono-energetic proton and alpha particle beams with energies ranging from $0.1 \mathrm{MeV}$ to $20 \mathrm{MeV}$ using $\mathrm{C}_{3} \mathrm{H}_{8}$ and $\mathrm{N}_{2}$ as target gases. These measured distributions were then compared to those obtained from simulations carried out with PTra. Due to the limitations of the vacuum system and the vacuum measuring system, the maximum pressure which can be applied in the ionisation volume is 1.2 mbar. According to the mean free path calculated from cross sections used in PTra [20,21], equivalent cluster size distributions for protons and alpha particles in both media should be obtained for pressures of 0.34 mbar $\mathrm{C}_{3} \mathrm{H}_{8}$ and 1.2 mbar $\mathrm{N}_{2}$. However, since the spatial distribution of the extraction efficiency (see Fig. 7) changes such that with increasing pressure of the target gas, the extraction efficiency increases for points of ionisation events having the same height above the extraction aperture. Therefore, for $\mathrm{C}_{3} \mathrm{H}_{8}$ the pressure has to be increased in order to obtain cluster size distributions equivalent to those measured for $1.2 \mathrm{mbar} \mathrm{N}_{2}$. This can be seen in Figure 8, where experimental data of the mean ionisation cluster size $M_{1}(p)$ for ${ }^{241} \mathrm{Am}$ alpha particles as a function of the pressure reveal, that a pressure of about 0.46 mbar for $\mathrm{C}_{3} \mathrm{H}_{8}$ yields the same mean ionisation cluster size as 1.2 mbar $\mathrm{N}_{2}$. This is in accordance with the extraction efficiency $\eta$ integrated along the particle track between the radius $r= \pm 2 \mathrm{~mm}$ at a height $h=16.25 \mathrm{~mm}$ (i.e. $\left.\eta=\int \eta(h=16.25 \mathrm{~mm}, p) d r\right)$, which is 0.23 for 1.2 mbar $\mathrm{N}_{2}$ and 0.17 for 0.46 mbar $\mathrm{C}_{3} \mathrm{H}_{8}$. For identical $\eta$, identical $M_{1}(p)$ would be achieved for $1.2 \mathrm{mbar}$ $\mathrm{N}_{2}$ and 0.34 mbar $\mathrm{C}_{3} \mathrm{H}_{8}$, however, for the aforementioned values of $\eta$, identical $M_{1}(p)$ are expected for 1.2 mbar $\mathrm{N}_{2}$ and $(0.23 / 0.17) 0.34$ mbar $=0.46$ mbar $\mathrm{C}_{3} \mathrm{H}_{8}$.

\section{Comparison of measured and simulated data}

The simulated ionisation cluster size distributions were obtained using the PTra Monte Carlo code. The code and the models, which it contains, were described in references $[3,21]$, and in particular the cross sections implemented in the code and the extensions to the models to simulate the radiation transport in the vicinity of the Bragg peak were discussed extensively in reference [21].

Measured and simulated mean ionisation cluster sizes $M_{1}(Q)$ for protons of different kinetic energy $T$ in $1.2 \mathrm{mbar}$ $\mathrm{N}_{2}$ and 1.2 mbar $\mathrm{C}_{3} \mathrm{H}_{8}$ have been compared by Bug et al. [21]. Further comparison appears in Figures 10 and 11 of this present work for measured and simulated ionisation cluster size distributions $P_{\nu}(Q)$ for protons and alpha particles of two different energies in 1.2 mbar $\mathrm{N}_{2}$ and 1.2 mbar $\mathrm{C}_{3} \mathrm{H}_{8}$. For both types of primary particles and for both gases, the measurements and simulations agree well for small ionisation clusters having a high probability of occurrence, which dominate both the mean and distribution of ionisation cluster sizes. The agreement is noticeably less for larger ionisation clusters which occur with low probability, thereby rendering them more susceptible to background effects (i.e. electronic noise and/or additional secondary ions). This discrepancy between measured and simulated large cluster sizes is more pronounced for $\mathrm{C}_{3} \mathrm{H}_{8}$ than for $\mathrm{N}_{2}$.

In Figure 12, the measured and simulated mean ionisation cluster sizes $M_{1}(T)$ for protons and alpha particles of different kinetic energy $T$ measured in $\mathrm{N}_{2}$ and $\mathrm{C}_{3} \mathrm{H}_{8}$ are shown. For the measurements, the pressures were chosen according to those which yielded the best agreement in the mean ionisation cluster size for the two target gases, namely 1.2 mbar for $\mathrm{N}_{2}$ and 0.46 mbar for $\mathrm{C}_{3} \mathrm{H}_{8}$ 

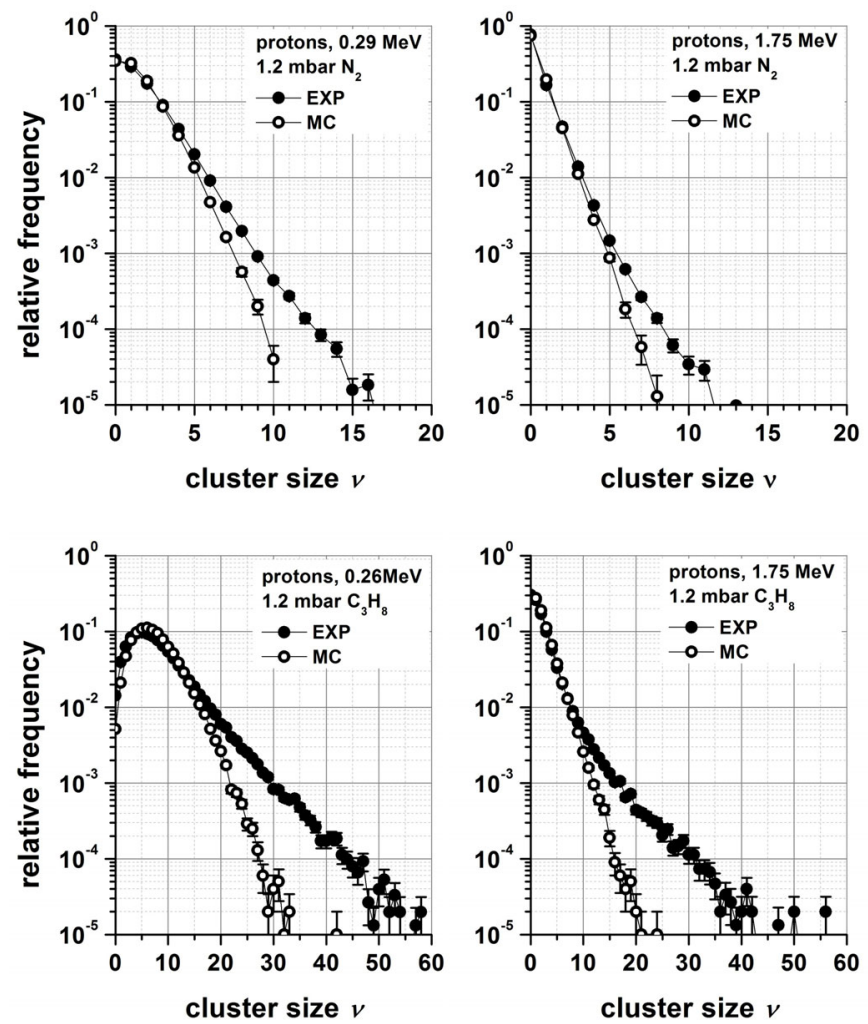

Fig. 10. Measured (EXP) and Monte Carlo simulated (MC) ionisation cluster size distributions for protons of two different energies in 1.2 mbar $\mathrm{N}_{2}$ (top) and 1.2 mbar $\mathrm{C}_{3} \mathrm{H}_{8}$ (bottom).

in case of alpha particles and 0.425 mbar for protons. For both primary particle types, the mean ionisation cluster sizes show the same energy dependence. For protons, the mean ionisation cluster sizes agree with each other within the experimental uncertainties over the entire energy range investigated. For alpha particles with energies above about $0.7 \mathrm{MeV}$, which is just higher than the Bragg peak energy, the measured and simulated mean ionisation cluster sizes generally agree well within the experimental uncertainties. Below this energy, the simulated data deviate from the measured values by as much as $25 \%$ which is more than the experimental uncertainties. This deviation may be due to the poor knowledge of the ionisation and charge transfer cross sections used in the simulations for energies around and lower than the Bragg peak energy [21].

Ionisation cluster size distributions for protons and alpha particles of different kinetic energy in 1.2 mbar $\mathrm{N}_{2}$ and 0.425 mbar $\mathrm{C}_{3} \mathrm{H}_{8}$ (protons) and in 1.2 mbar $\mathrm{N}_{2}$ and 0.46 mbar $\mathrm{C}_{3} \mathrm{H}_{8}$ (alpha particles) are plotted in Figure 13. The cluster sizes for the different radiation qualities $Q$ (i.e. protons and alpha particles) show almost identical distributions $P_{\nu}(Q)$ for the different combinations of gases and pressures, when considering measured and simulated ionisation cluster size distributions separately. However, the deviation between measured and simulated values still exists for larger ionisation clusters.
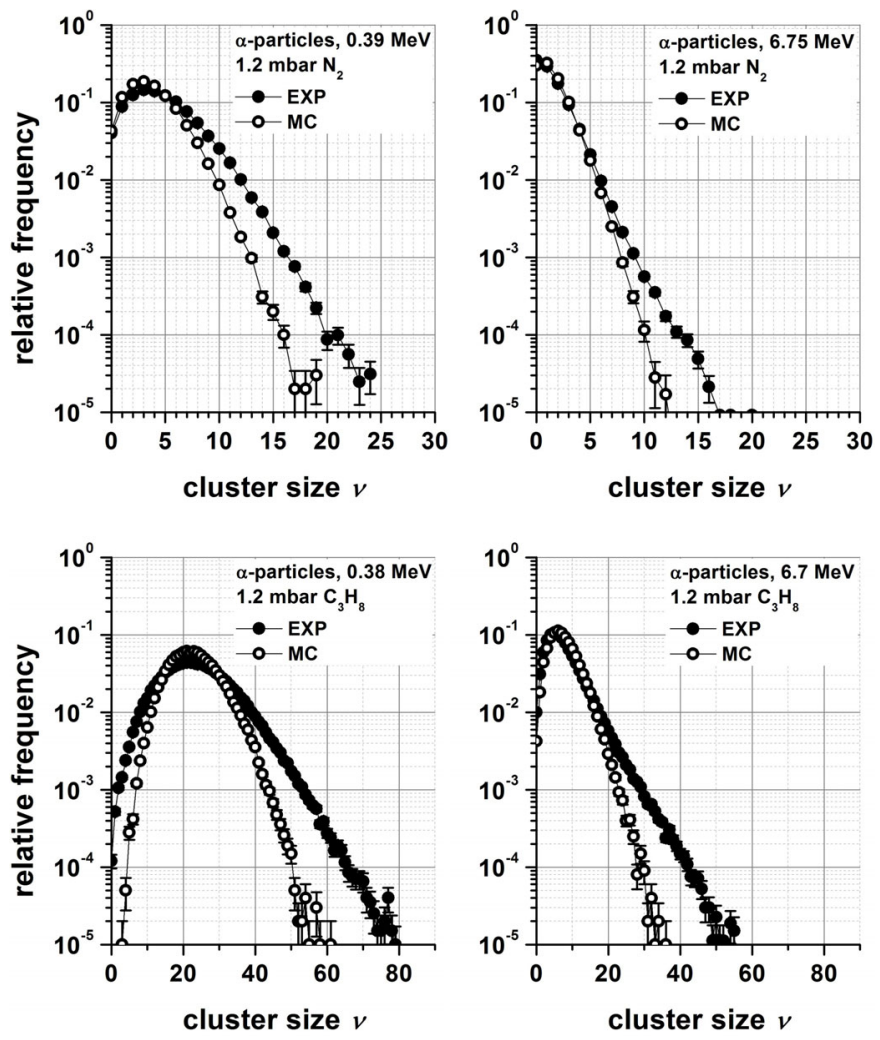

Fig. 11. Measured (EXP) and Monte Carlo simulated (MC) ionisation cluster size distributions for alpha particles of two different energies in 1.2 mbar $\mathrm{N}_{2}$ (top) and 1.2 mbar $^{\mathrm{C}_{3} \mathrm{H}_{8}}$ (bottom).
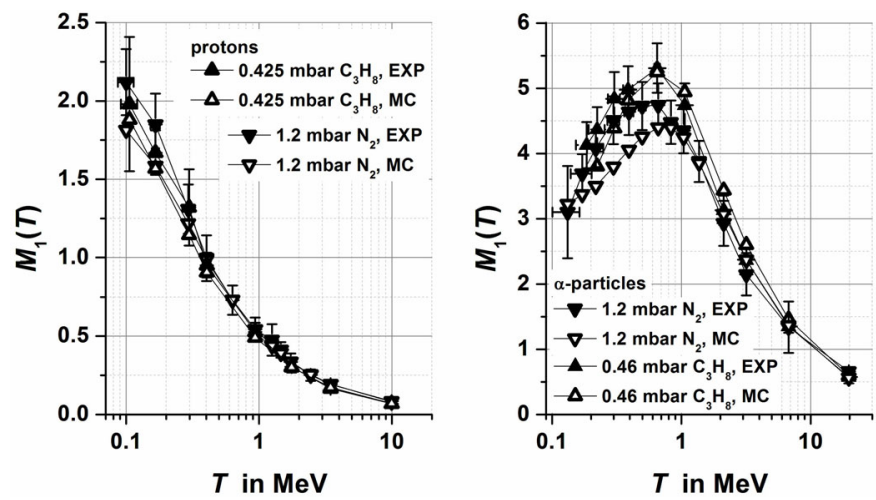

Fig. 12. Measured (EXP) and Monte Carlo simulated (MC) mean ionisation cluster size $M_{1}(T)$ for protons (left) and alpha particles (right) of different kinetic energy $T$ in 1.2 mbar $\mathrm{N}_{2}$ and 0.425 mbar $\mathrm{C}_{3} \mathrm{H}_{8}$ (protons) and 0.46 mbar $\mathrm{C}_{3} \mathrm{H}_{8}$ (alpha particles).

\section{Effect of secondary ionisations in the comparison of measured and simulated data}

As mentioned earlier, during the transport of ions created by the primary ionising particle, secondary ions are produced within the ion transport optics due to scattering downstream of the extraction aperture. The simulations, 

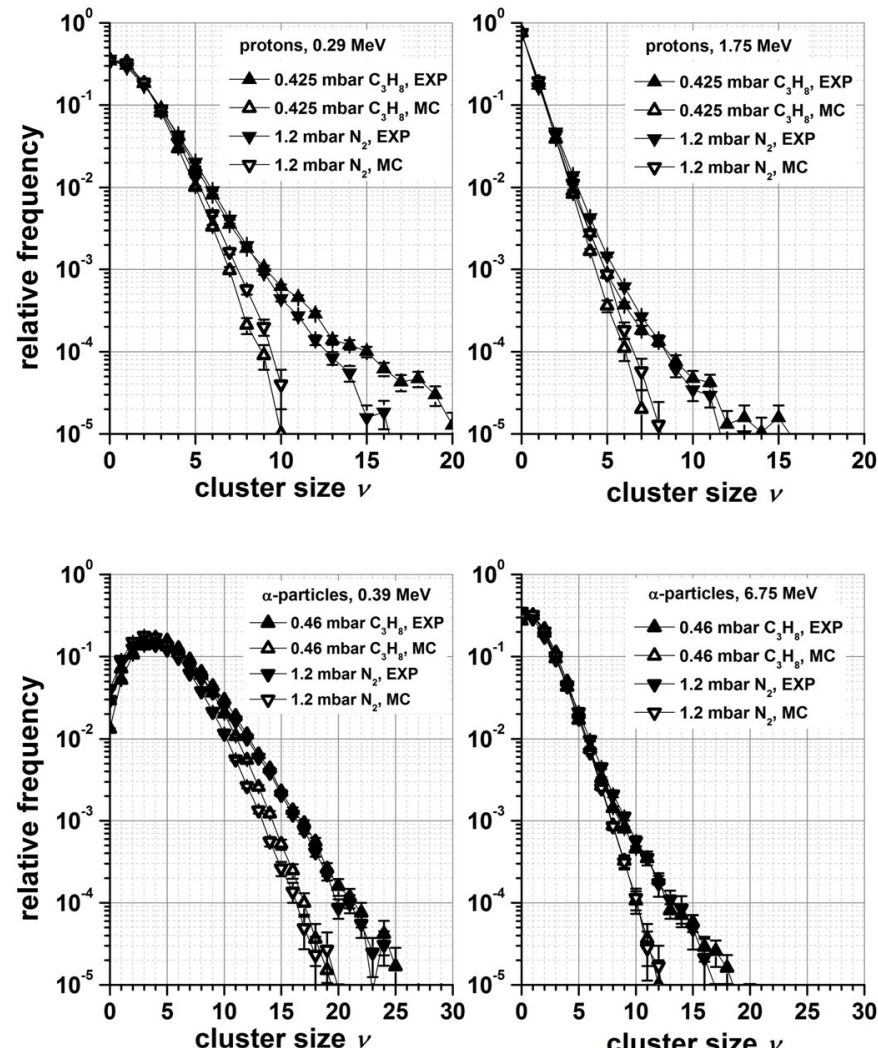

Fig. 13. Measured (EXP) and Monte Carlo simulated (MC) ionisation cluster size distributions for protons (top) and alpha particles (bottom) of different kinetic energy in 1.2 mbar $\mathrm{N}_{2}$ and 0.425 mbar $\mathrm{C}_{3} \mathrm{H}_{8}$ (protons) and $1.2 \mathrm{mbar}_{2}$ and 0.46 mbar $\mathrm{C}_{3} \mathrm{H}_{8}$ (alpha particles).

however, do not include these secondary ions. For the purpose of comparison with measured data, the simulated ionisation cluster size distributions need to be adjusted by taking into account these secondary ionisations.

The inclusion of secondary ionisations into the simulated ionisation cluster size distributions is a multi-step process. The first step is to split the simulated cluster size distribution into two distributions according to the probability $\varepsilon$ for an ionised gas molecule from an ionisation cluster to hit the cone-shaped electrode after passing the extraction aperture. The first distribution represents the ions which reach the secondary electron multiplier directly without being scattered. The second one represents those ions which are scattered in the jet of neutral gas molecules downstream of the extraction aperture and which consequently hit the cone-shaped electrode. The latter distribution is, in the next step, convoluted with a Poisson distribution using an assumed expectation value $\lambda$ representing the secondary ions created by a single ionised target gas molecule hitting the cone-shaped electrode. The result of this convolution describes the total distribution of secondary ionisations. Since the secondary ions are created in the vicinity of the extraction aperture, it has to be taken into account, that these secondary ions also can be scattered by neutral gas atoms downstream of the extraction aperture and have a probability $\varepsilon$ of striking the coneshaped electrode. The distribution resulting from the convolution itself has to therefore undergo the same process of splitting and convolution as described above. This iterative procedure is continued until convergence is reached, that is until the increase in the amount of secondary ionisations becomes insignificant. Finally, the distributions from the splitting processes of all iterations representing the ions reaching the secondary electron multiplier without scattering and the total distribution of secondary ions of the last iteration step are convoluted. At the conclusion of this process, the simulated distribution includes the contribution of secondary ionisations, which can now be compared with the measured data.

In order to quantify the degree of agreement between measured and simulated data, a quantity $R(Q)$ was applied, which is defined as follows:

$$
R(Q)=\frac{1}{n} \sum_{\nu=0}^{\infty} \frac{\left|P_{\nu}^{\mathrm{EXP}}(Q)-P_{\nu}^{\mathrm{MC}+\mathrm{SEC}}(Q)\right|}{P_{\nu}^{\mathrm{EXP}}(Q)+P_{\nu}^{\mathrm{MC}+\operatorname{SEC}}(Q)} W_{\nu}^{\mathrm{EXP}},
$$

where $P_{\nu}^{\mathrm{EXP}}(Q)$ and $P_{\nu}^{\mathrm{MC}+\operatorname{SEC}}(Q)$ are the measured and background-corrected simulated data, respectively. The sum includes only those summands where both $P_{\nu}^{\mathrm{EXP}}(Q)>0$ and $P_{\nu}^{\mathrm{MC}+\mathrm{SEC}}(Q)>0$, and $n$ is the number of data points, for which both $P_{\nu}^{\mathrm{EXP}}(Q)>0$ and $P_{\nu}^{\mathrm{MC}+\operatorname{SEC}}(Q)>0 . W_{\nu}^{\mathrm{EXP}}$ describes the weight of the corresponding summand which is defined as follows:

$$
W_{\nu}^{\mathrm{EXP}}=1-\frac{1}{\sqrt{N_{\nu}^{\mathrm{EXP}}}} \quad \text { if } \quad N_{\nu}^{\mathrm{EXP}}>0,
$$

where $N_{\nu}^{\mathrm{EXP}}$ is the number of measured ionisation clusters of size $\nu$. As previously mentioned, for a given target gas at a given pressure, the two parameters $\lambda$ and $\varepsilon$ describing the background of secondary ions, and therefore also the position of the minimum of $R$ as a function of $\lambda$ and $\varepsilon$, must be identical for each ionisation cluster size distribution measured with primary particles of any radiation quality for a specific gas target.

Figure 14 shows the contour plots for $R$, which is the mean of $R(Q)$, averaged over the $R(Q)$ values of all energies for a given particle type and a given target, as a function of $\lambda$ and $\varepsilon$ for the data sets obtained with 1.2 mbar of the target gases $\mathrm{N}_{2}$ (left column) and $\mathrm{C}_{3} \mathrm{H}_{8}$ (middle column) for protons (top row), alpha particles (middle row) and for the combination of both particle types (bottom row). In addition, $R$ is plotted for the data set obtained with protons in 0.425 mbar $\mathrm{C}_{3} \mathrm{H}_{8}$, alpha particles in 0.46 mbar $\mathrm{C}_{3} \mathrm{H}_{8}$ and the combination of the two (right column).

For all data sets, the minimum of $R$ stretches along a long and narrow valley whose length differs between $\mathrm{N}_{2}$ and $\mathrm{C}_{3} \mathrm{H}_{8}$ (note the different scaling on the $\varepsilon$-axis for $\mathrm{N}_{2}$ and $\mathrm{C}_{3} \mathrm{H}_{8}$ ). Furthermore, the effect of changing the pressure of $\mathrm{C}_{3} \mathrm{H}_{8}$ from 1.2 mbar to 0.46 mbar or 0.425 mbar is practically negligible. As expected, the minima of $R$ for the data sets of protons and alpha particles for both the same target gas and pressure almost coincide. It is therefore sufficiently justified to combine the two data sets for 

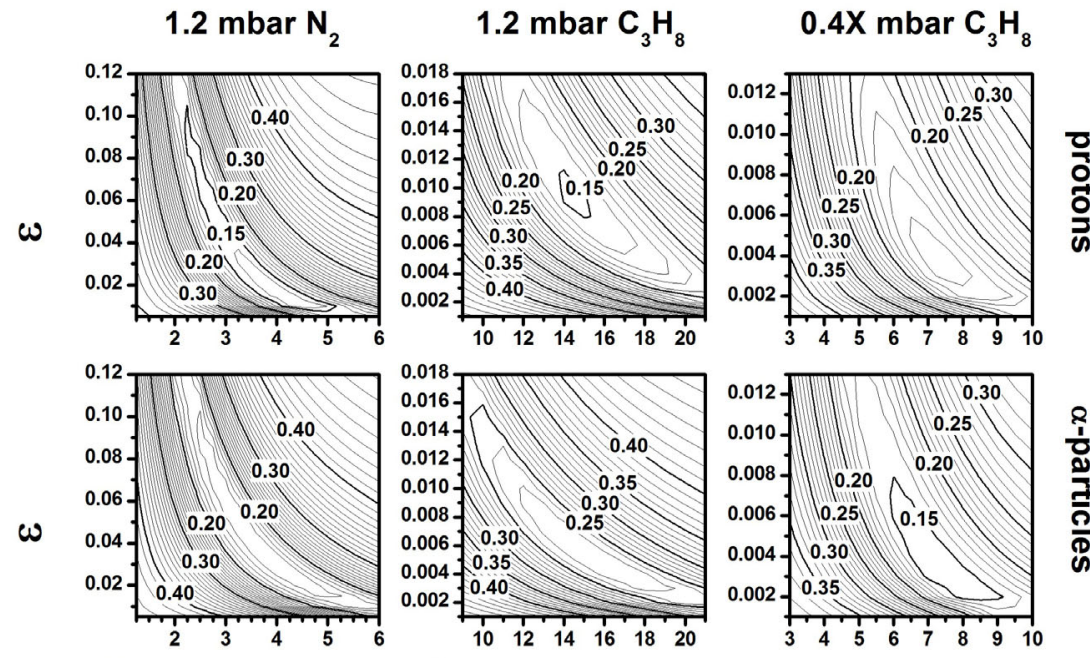

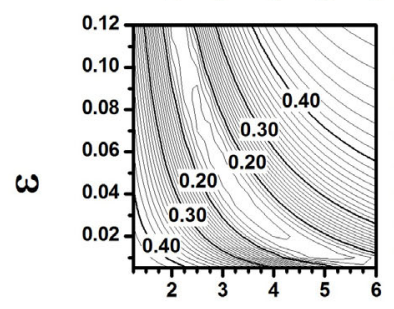

$\lambda$

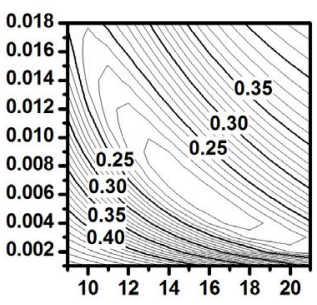

$\lambda$

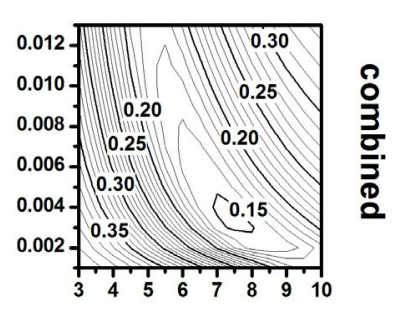

$\lambda$

Fig. 14. Contour plots for $R$ as a function of expectation value $\lambda$ and probability $\varepsilon$ for the data sets obtained with 1.2 mbar $\mathrm{N}_{2}$ (left column) and 1.2 mbar $\mathrm{C}_{3} \mathrm{H}_{8}$ (middle column) for protons (top row) and alpha particles (middle row) and for the combination of both particle types (bottom row). Also shown are the data set obtained with protons in $0.425 \mathrm{mbar}_{3} \mathrm{H}_{8}$, alpha particles in 0.46 mbar $\mathrm{C}_{3} \mathrm{H}_{8}$ and the combination of the two (right column).
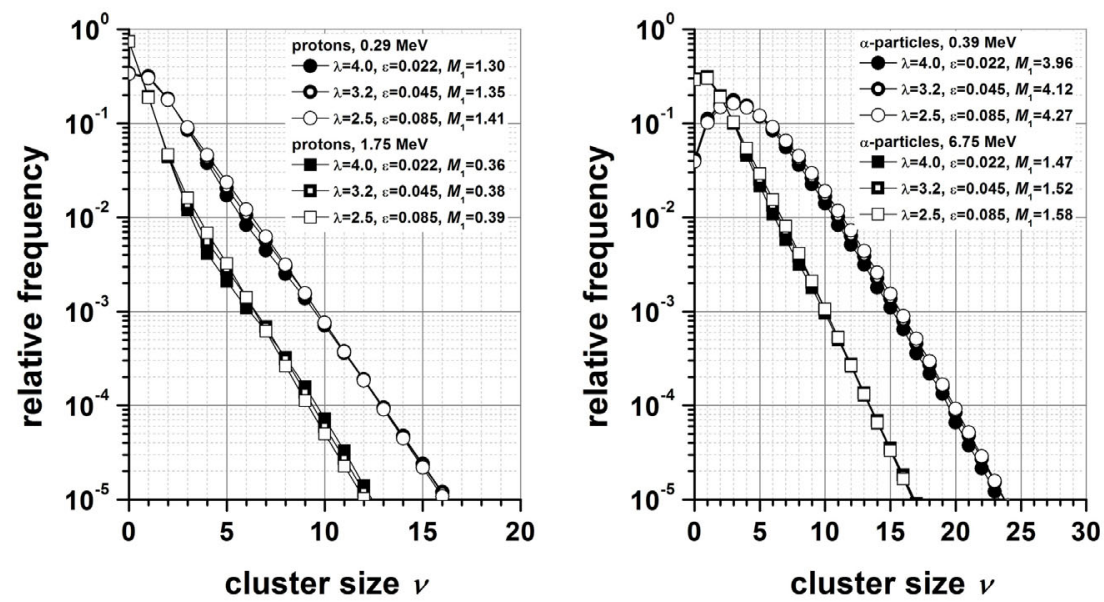

Fig. 15. Simulated background-corrected ionisation cluster size distributions $P_{\nu}(Q)$ for protons (left) and alpha particles (right) of different kinetic energy in 1.2 mbar $\mathrm{N}_{2}$. The background correction was carried out for different $\lambda$ and $\varepsilon$ values as given in the legend, which also includes the resulting mean ionisation cluster size $M_{1}$.

determining $\lambda$ and $\varepsilon$ for a common minimum of $R$. The valley bottom is orientated in such a way that an increase in $\lambda$ corresponds with a decrease in $\varepsilon$ and vice versa. Thus, a deviation of one of the two parameters from the value leading to a minimum of $R$ can be compensated to some extent by a deviation of the other parameter in the opposite direction. This compensation effect is shown in Figure 15 for protons (left) and alpha-particles (right) in 1.2 mbar $\mathrm{N}_{2}$.
Each plot shows simulated ionisation cluster size distributions calculated for two different primary particle energies, where each cluster size distribution has been background corrected with respect to secondary ions using different combinations of parameters $\lambda$ and $\varepsilon$. The three combinations of $\lambda$ and $\varepsilon$ were chosen to be located at the centre and either end of the valley describing the minimum values of $R$. For all three combinations of $\lambda$ and $\varepsilon$, the simulated 

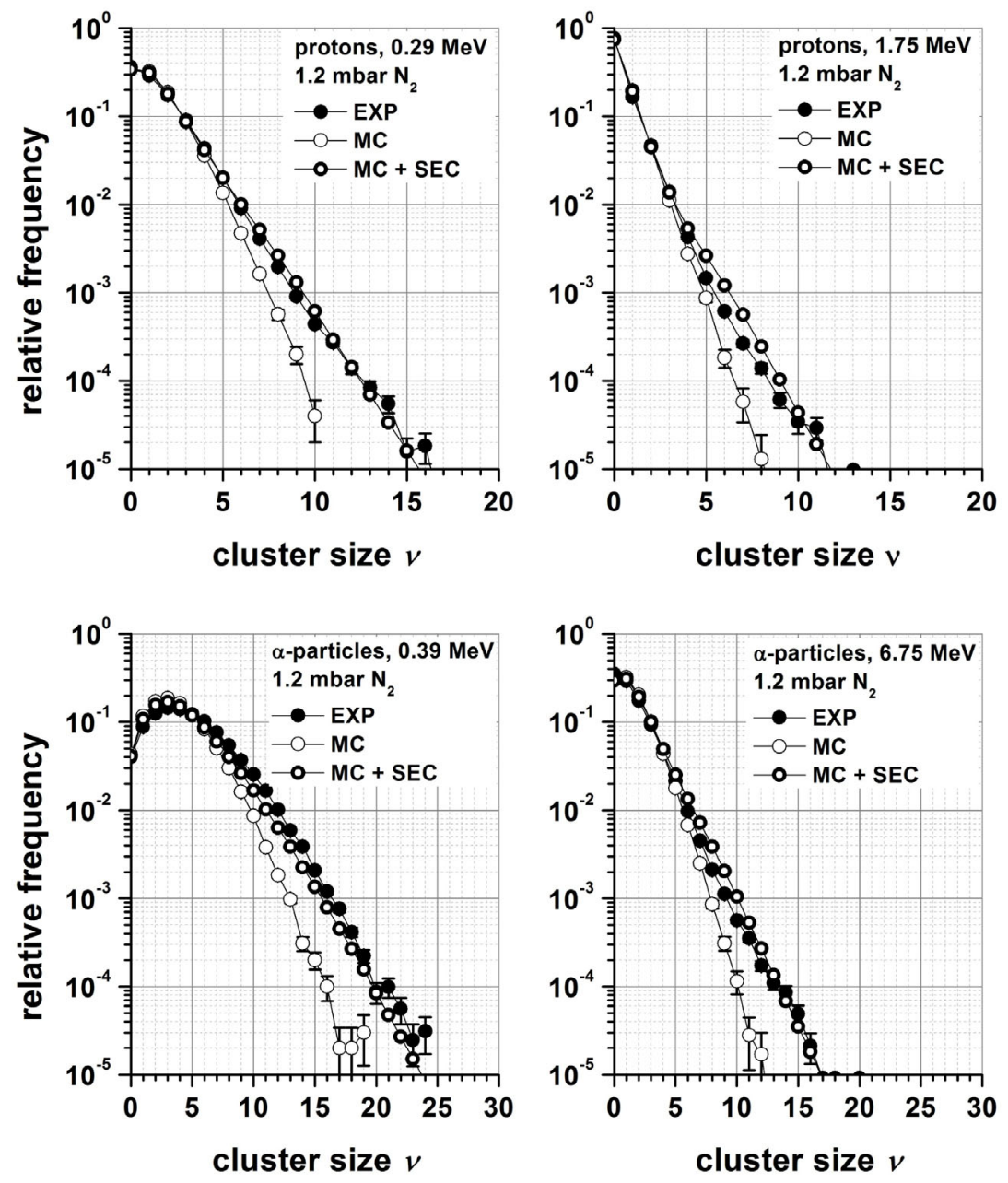

Fig. 16. Ionisation cluster size distributions measured (EXP) and Monte Carlo simulated with and without background correction (MC and MC + SEC, respectively) for protons (top) and alpha particles (bottom) of two different energies in 1.2 mbar $\mathrm{N}_{2}$.

and background-corrected cluster size distributions are almost identical and the mean cluster sizes $M_{1}$ show only slight differences. Therefore, the following discussion will be limited to the simulated cluster size distributions which have been background-corrected with the $\lambda$ and $\varepsilon$ parameter set located in the centre of the valley denoting the minima of $R$ (i.e. $\lambda=3.2$ and $\varepsilon=0.045$ for 1.2 mbar $\mathrm{N}_{2}$; $\lambda=15$ and $\varepsilon=0.0065$ for 1.2 mbar $\mathrm{C}_{3} \mathrm{H}_{8}$; and $\lambda=7.5$

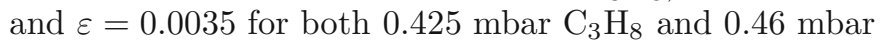
$\left.\mathrm{C}_{3} \mathrm{H}_{8}\right)$.

The comparison of measured ionisation cluster size distributions with those simulated with and without background correction is shown in Figure 16 for protons (top) and alpha particles (bottom) of two different energies in 1.2 mbar $\mathrm{N}_{2}$. Due to the small value of $\varepsilon=0.045$, the main contribution of secondary ions is to larger ionisation clusters. In all four cases, the simulated background-corrected cluster size distributions agree significantly better with the measured data than the corresponding simulated "uncorrected" distributions which have not been subject to background-correction. Since the background correction almost only contributes to the larger clusters which have a low probability of occurrence, its effect on the mean cluster sizes $M_{1}(T)$ is comparatively small (see Fig. 17), resulting in an increase of only a few percent.

The comparison of measured ionisation cluster size distributions with those simulated with and without background correction for two different energies of protons (top) and alpha particles (bottom) in 1.2 mbar $\mathrm{C}_{3} \mathrm{H}_{8}$ shows Figure 18. When compared to the corresponding distributions in 1.2 mbar $\mathrm{N}_{2}$ (see Fig. 16), the contribution of secondary ions is more pronounced in 1.2 mbar $\mathrm{C}_{3} \mathrm{H}_{8}$ than in 1.2 mbar $\mathrm{N}_{2}$, despite the value of $\varepsilon$ being significantly smaller (i.e. 0.0065 and 0.045 in $\mathrm{C}_{3} \mathrm{H}_{8}$ and $\mathrm{N}_{2}$, respectively). This is due to the larger value of $\lambda=15$ for 1.2 mbar $\mathrm{C}_{3} \mathrm{H}_{8}$ compared to $\lambda=3.2$ for 1.2 mbar $\mathrm{N}_{2}$, and hence, a greater number of large background clusters in the distribution of secondary ions. Consequently, a greater increase in the amount of large ionisation clusters is observed for 1.2 mbar $\mathrm{C}_{3} \mathrm{H}_{8}$ than for 1.2 mbar $\mathrm{N}_{2}$.

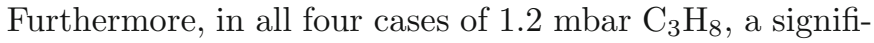
cantly better agreement can be seen between the measured and simulated background-corrected cluster size distributions than for the measured and simulated uncorrected cluster size distributions. However, at small cluster sizes 

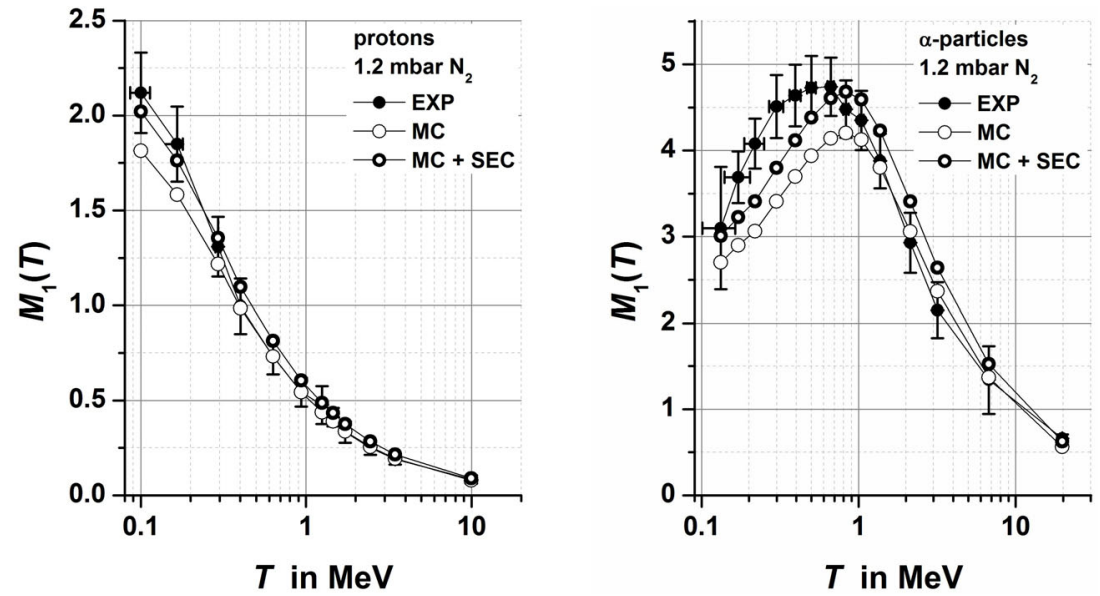

Fig. 17. Mean ionisation cluster size $M_{1}(T)$ measured (EXP) and Monte Carlo simulated with and without background correction (MC and MC + SEC, respectively) for protons (left) and alpha particles (right) of different kinetic energy $T$ in $1.2 \mathrm{mbar} \mathrm{N}_{2}$.

well below the mean, the deviation between measured and simulated background-corrected cluster size distributions for $0.38 \mathrm{MeV}$ alpha particles in $1.2 \mathrm{mbar} \mathrm{C}_{3} \mathrm{H}_{8}$ persists and cannot be explained by a background of secondary ions. Also, the effect of the background correction on the mean cluster sizes $M_{1}(T)$ is comparatively small (see Fig. 19). While the increase in mean cluster sizes for 1.2 mbar $_{3} \mathrm{H}_{8}$ is somewhat larger than that for $1.2 \mathrm{mbar}_{2}$, it is of the same order of magnitude, namely a few percent.

Figure 20 compares measured ionisation cluster size distributions with those simulated with and without background-correction for two different energies of protons in 0.425 mbar $\mathrm{C}_{3} \mathrm{H}_{8}$ (top) and alpha particles in 0.46 mbar $\mathrm{C}_{3} \mathrm{H}_{8}$ (bottom). Since both $\varepsilon$ and $\lambda$ parameters describing the contribution of secondary ions are lower for 0.425 mbar and 0.46 mbar $\mathrm{C}_{3} \mathrm{H}_{8}(\varepsilon=0.0035$ and $\lambda=7.5)$ than for 1.2 mbar $\mathrm{C}_{3} \mathrm{H}_{8}(\varepsilon=0.0065$ and $\lambda=15)$, the contribution of the secondary ions in 0.425 mbar and 0.46 mbar $\mathrm{C}_{3} \mathrm{H}_{8}$ is less pronounced than that for 1.2 mbar $\mathrm{C}_{3} \mathrm{H}_{8}$. In all four cases, as seen in Figure 18, the agreement between measured ionisation cluster size distributions and those simulated with background correction is significantly better than the agreement between measured and simulated uncorrected cluster size distribution. Again, the effect of the background correction on the mean cluster sizes $M_{1}(T)$ is small (see Fig. 21). The increase in mean cluster sizes for 0.425 mbar and $0.46 \mathrm{mbar}_{3} \mathrm{C}_{3} \mathrm{H}_{8}$ is smaller

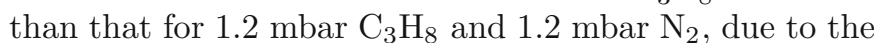
smaller contribution of the secondary ions.

Finally, as discussed earlier, the two parameters describing the secondary ionisations, $\lambda$ and $\varepsilon$, were determined by minimising the deviation between the measured and simulated background-corrected cluster size distributions. Checking the plausibility of $\varepsilon$, which is a parameter determined by ion transport properties in the ion optics, is not feasible. $\lambda$, on the other hand, is primarily determined by the ionisation cross sections for electrons and the target gas density downstream of the extraction aperture. Assuming similar characteristics for 1.2 mbar $\mathrm{C}_{3} \mathrm{H}_{8}$ and 1.2 mbar $\mathrm{N}_{2}$, with respect to the gas density for the effusive gas jet downstream of the extraction aperture, the difference in $\lambda$ should be dominated by the difference in the ionisation cross sections for electrons. For $2.5 \mathrm{keV}$ electrons, which are responsible for the aforementioned production of secondary ions, the ratio of ionisation cross sections of $\mathrm{C}_{3} \mathrm{H}_{8}$ and $\mathrm{N}_{2}$ as used in PTra [21] is $\sigma_{\mathrm{C}_{3} \mathrm{H}_{8}} / \sigma_{\mathrm{N}_{2}} \cong 3.4$, resulting in $\lambda$ values of 10.9 and 3.2 for $1.2 \mathrm{mbar}_{3} \mathrm{H}_{8}$ and $1.2 \mathrm{mbar}_{2}$, respectively. The $\lambda$ value for $\mathrm{C}_{3} \mathrm{H}_{8}$ agrees quite reasonably with that of $\lambda=15$ previously determined for 1.2 mbar $\mathrm{C}_{3} \mathrm{H}_{8}$. The difference in $\lambda$ between 1.2 mbar and 0.425 or 0.46 mbar $\mathrm{C}_{3} \mathrm{H}_{8}$ should correspond to the ratio of different gas densities, which in turn should correspond to the different gas pressures. With a ratio of 2.7 for the different gas pressures, the value of $\lambda$ for 0.443 and 1.2 mbar $\mathrm{C}_{3} \mathrm{H}_{8}$ becomes 7.5 and 20.3, respectively, which also agrees reasonably with the previously determined value of $\lambda=15$ for 1.2 mbar $\mathrm{C}_{3} \mathrm{H}_{8}$. Thus, the ratio of different $\lambda$ values obtained by minimising the deviation between measured and simulated background-corrected data correspond quite well with those obtained using the ionisation cross sections for electrons and the target gas densities.

\section{Conclusions}

Measured and simulated ionisation cluster size distributions $P_{\nu}(Q)$ for protons and alpha particles of different energies in 1.2 mbar $\mathrm{N}_{2}, 0.425$ mbar $\mathrm{C}_{3} \mathrm{H}_{8}$ (protons only),

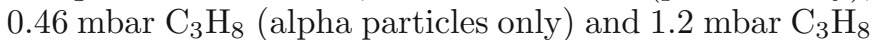
were compared in order to benchmark the PTra Monte Carlo code. To the best of our knowledge, a comparison of both, in such detail and in an energy range extending down to an energy close to the Bragg peak energy in the case of protons and down to energies even lower than the Bragg peak energy in the case of alpha particles, was carried out for the first time. Furthermore, the background of secondary ionisations, which are produced in the ion optics, for the first time is taken into account explicitly in this comparison. 

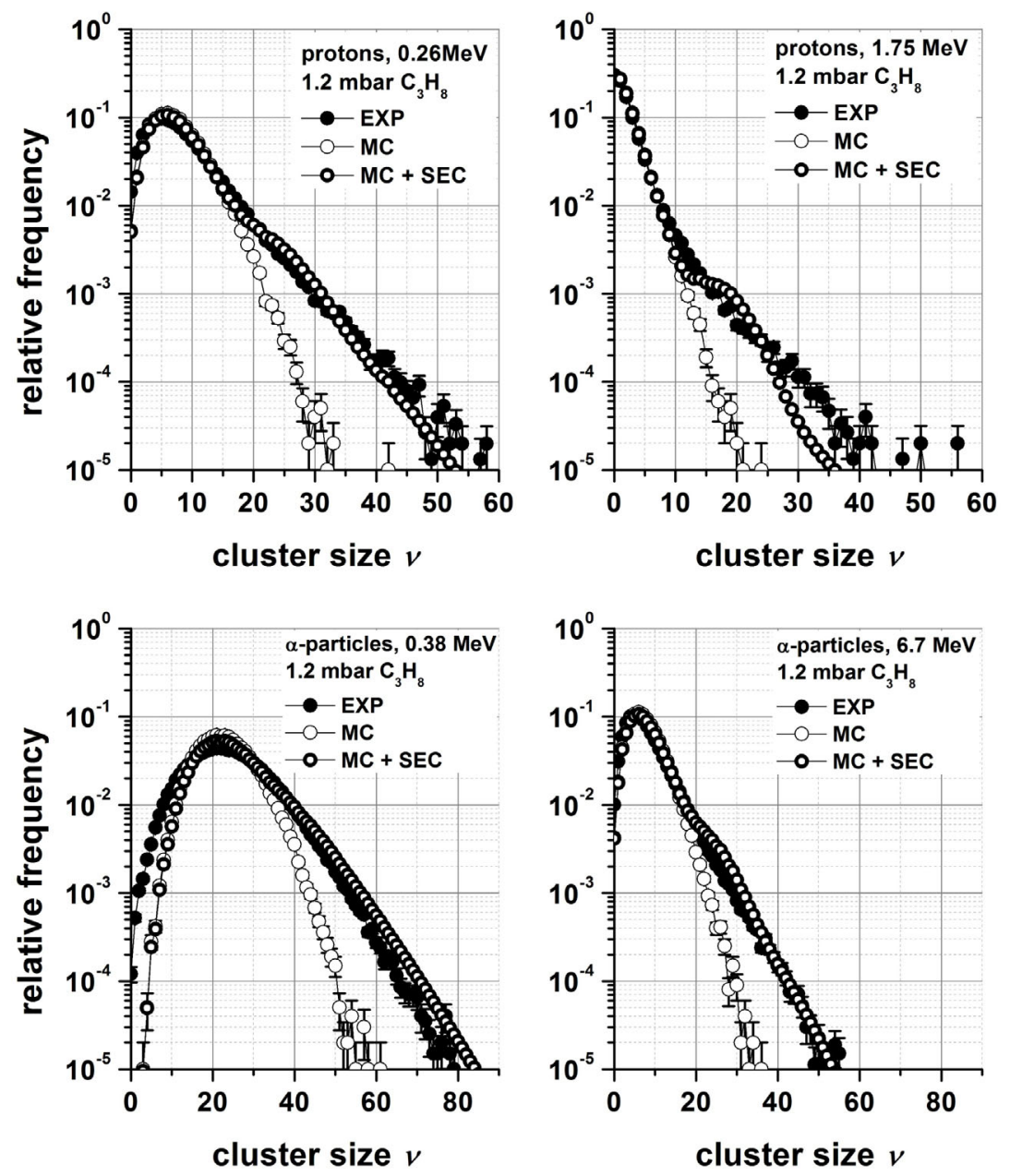

Fig. 18. Ionisation cluster size distributions measured (EXP) and Monte Carlo simulated with and without background correction ( $\mathrm{MC}$ and $\mathrm{MC}+\mathrm{SEC}$, respectively) for protons (top) and alpha particles (bottom) of two different energies in $1.2 \mathrm{mbar} \mathrm{C}_{3} \mathrm{H}_{8}$.

A good agreement, well within the experimental uncertainties, was observed for mean ionisation cluster sizes $M_{1}(T)$ of alpha particles with energies greater than about $0.7 \mathrm{MeV}$, which is just higher than the Bragg peak energy. For energies around and lower than the Bragg peak energy, the simulated data deviate by up to $25 \%$ from the measured values by more than the experimental uncertainties. The agreement between measured and simulated mean ionisation cluster sizes $M_{1}(T)$ was better for protons than for alpha particles. Measured and simulated cluster size distributions $P_{\nu}(Q)$ for protons and alpha particles of different energies agree well for small ionisation clusters, which have a high probability of occurrence. Significant deviations are only observed for large ionisation cluster sizes. These deviations can be explained by a background of secondary ions, which are produced in the region downstream of the extraction aperture by $2.5 \mathrm{keV}$ electrons released by ions of the "primary" ionisation cluster hitting an electrode in the ion transport system. Including this background of secondary ions in the simulated cluster size distributions leads to a significantly better agreement between measured and simulated data. However, for small cluster sizes well below the mean, the deviation between measured and simulated background-corrected cluster size distributions persists and cannot be explained with a background of secondary ions, but may be attributed to the poor knowledge of ionisation and charge transfer cross sections for particles with low energies used in the simulations.

Since the production of secondary ions depends on the effusive gas jet properties as well as the geometry and potentials of the ion optics downstream of the extraction aperture, the relative contribution of the secondary ion background is independent of the creation of the ionisation cluster and therefore independent of the radiation quality of the primary particle. Thus, each of the two parameters $\lambda$ and $\varepsilon$, which describe the background of secondary ions, must have the same value for each cluster size distribution measured with primary particles of any radiation quality for a specific gas target. $\lambda$ and $\varepsilon$ were therefore determined by minimising the deviation between measured and simulated background-corrected cluster size distributions. Checking the plausibility of $\varepsilon$, which is a parameter determined by the ion transport properties in the ion optics, is not feasible. However, since $\lambda$ is mainly determined by the ionisation cross sections for electrons and the target 

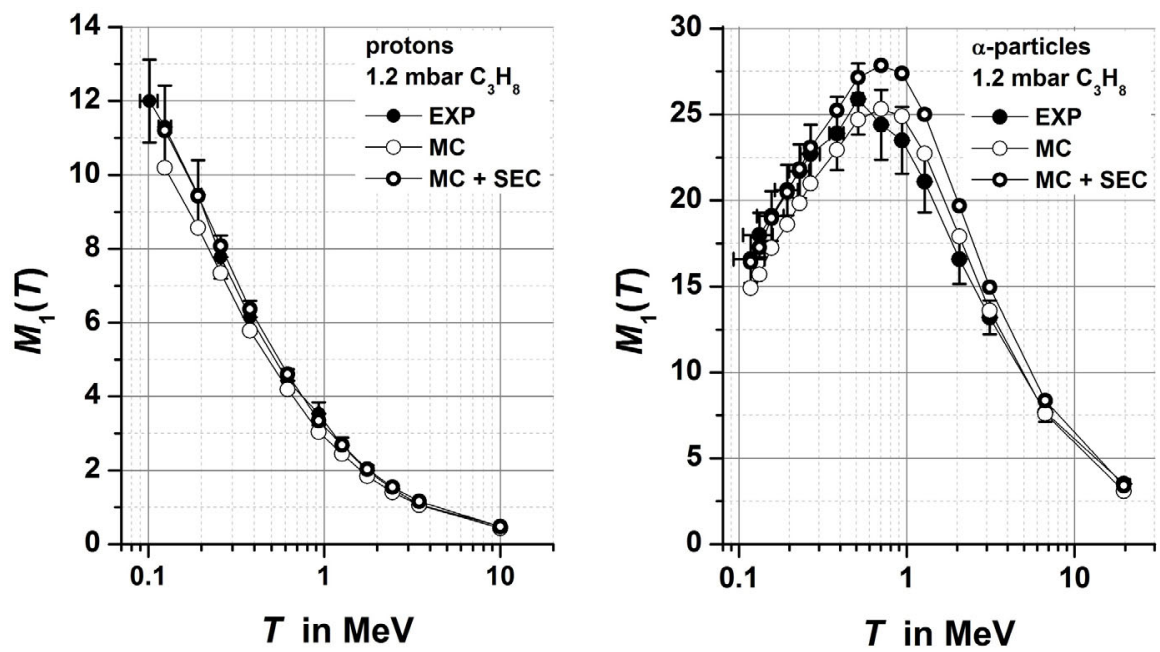

Fig. 19. Mean ionisation cluster size $M_{1}(T)$ measured (EXP) and Monte Carlo simulated with and without background correction ( $\mathrm{MC}$ and $\mathrm{MC}+\mathrm{SEC}$, respectively) for protons (left) and alpha particles (right) of different kinetic energy $T$ in 1.2 mbar $\mathrm{C}_{3} \mathrm{H}_{8}$.
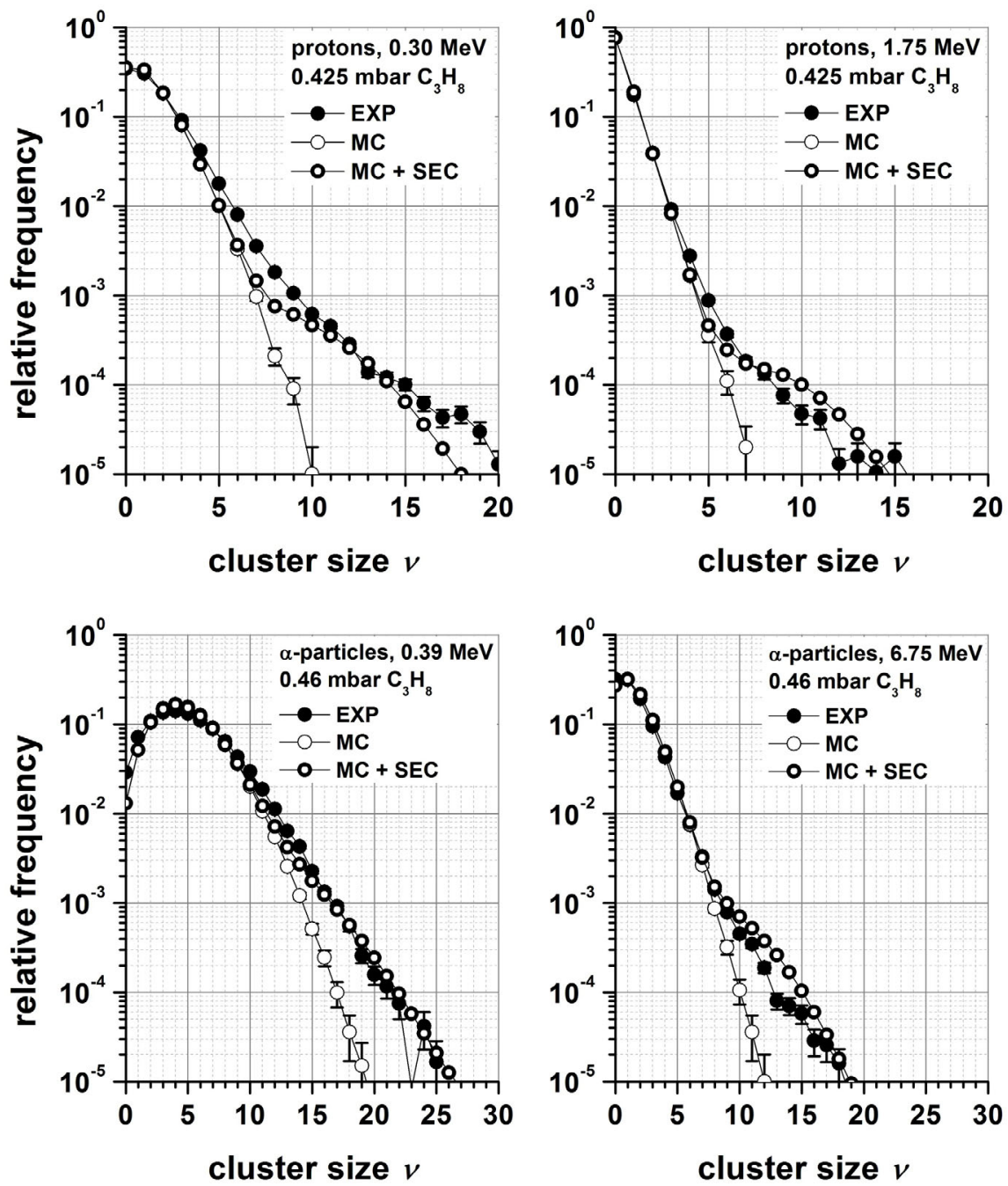

Fig. 20. Ionisation cluster size distributions measured (EXP) and Monte Carlo simulated with and without background correction ( $\mathrm{MC}$ and $\mathrm{MC}+\mathrm{SEC}$, respectively) for two different energies of protons in $0.425 \mathrm{mbar}_{3} \mathrm{H}_{8}$ (top) and of alpha particles in 0.46 mbar $\mathrm{C}_{3} \mathrm{H}_{8}$ (bottom). 

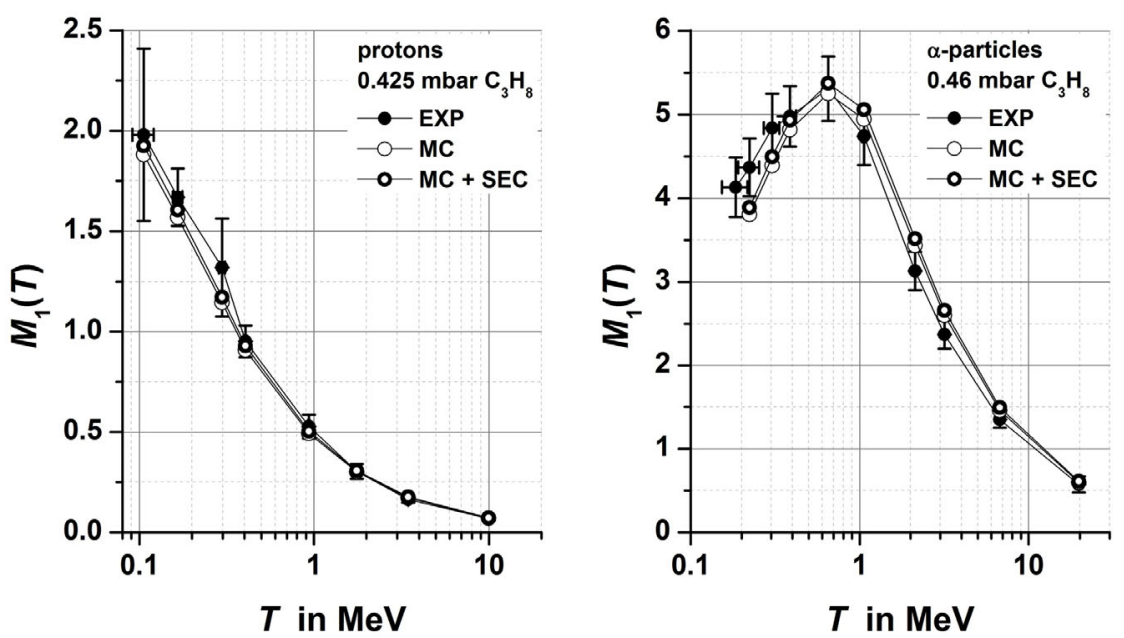

Fig. 21. Mean ionisation cluster size $M_{1}(T)$ measured (EXP) and Monte Carlo simulated with and without background correction ( $\mathrm{MC}$ and $\mathrm{MC}+\mathrm{SEC}$, respectively) for protons in 0.425 mbar $\mathrm{C}_{3} \mathrm{H}_{8}$ (left) and alpha particles in $0.46 \mathrm{mbar} \mathrm{C}_{3} \mathrm{H}_{8}$ (right) of different kinetic energies $T$.

gas densities downstream of the extraction aperture, the ratios of $\lambda$ values correspond quite reasonably with those of the ionisation cross sections for electrons and the target gas densities.

Basically, the quantity $R(Q)$ defined in equation (8) represents the average relative deviation of $P_{\nu}^{\mathrm{EXP}}(Q)$ and $P_{\nu}^{\mathrm{MC}+\operatorname{SEC}}(Q)$ from the arithmetic mean of $P_{\nu}^{\mathrm{EXP}}(Q)$ and $P_{\nu}^{\mathrm{MC}+\mathrm{SEC}}(Q)$, weighted with a function of the number of measured ionisation clusters of size $\nu$. However, due to its construction, the effect of the weighting function (Eq. (9)) is mostly limited to very small numbers of measured ionisation clusters at the large cluster size tail of the cluster size distribution. Therefore $R$, which is the mean of $R(Q)$, averaged over the $R(Q)$ values of all energies for a given particle type and a given target, can serve to some extend as a measure to quantitatively estimate the degree of agreement between measurement and simulation. Using the values of $R$ for the combination of $\lambda$ and $\varepsilon$ leading to the best agreement between measurement and simulation, it is found, that for all particle types and targets, the average relative deviation of $P_{\nu}^{\mathrm{EXP}}(Q)$ and $P_{\nu}^{\mathrm{MC}+\mathrm{SEC}}(Q)$ from their arithmetic mean ranges between $15 \%$ and $17 \%$, except for alpha particles in 1.2 mbar $\mathrm{C}_{3} \mathrm{H}_{8}$, where the average relative deviation of $P_{\nu}^{\mathrm{EXP}}(Q)$ and $P_{\nu}^{\mathrm{MC}+\mathrm{SEC}}(Q)$ from their arithmetic mean is about $23 \%$. In view of this overall good agreement between measured and simulated data, the PTra Monte Carlo code can be regarded as successfully benchmarked with respect to the models used in the simulation of the track structure of light ions in gaseous media and also with respect to the cross sections used for $\mathrm{N}_{2}$ and $\mathrm{C}_{3} \mathrm{H}_{8}$. The energy range of the ions, in which the PTra Monte Carlo code was benchmarked successfully, ranges down to energies close to the Bragg peak energy in the case of protons and down to energies even lower than the Bragg peak energy in the case of alpha particles.

For the comparison with measured cluster size distributions, the simulation results were corrected for the spatial distribution of the extraction probability and the production of secondary ions, which influence the measurements. While this procedure is justified for such a comparison, it cannot be applied in a situation when either cluster size distributions or derived quantities obtained from measurements are required. Instead, a deconvolution procedure needs to be applied to the measured data to remove the effects of the spatial distribution of the extraction efficiency and the background due to secondary ionisations. The development of such a procedure is the subject of further investigation.

The authors would like to thank the staff of the PTB Ion Accelerator Facility for their assistance and support during the measurements. The authors also express their gratitude to W. Helms, B. Lambertsen and A. Pausewang for their invaluable contributions to the preparation and carrying out of measurements and their assistance in the data processing. H. Nettelbeck's proofreading of the manuscript is also gratefully acknowledged.

\section{References}

1. D.T. Goodhead, J. Thacker, R. Cox, Int. J. Radiat. Biol. 63, 543 (1993)

2. D.T. Goodhead, Int. J. Radiat. Biol. 65, 7 (1994)

3. B. Grosswendt, Radiat. Environ. Biophys. 41, 103 (2002)

4. L. De Nardo, P. Colautti, V. Conte, W.Y. Baek, B. Großwendt, G. Tornielli, Radiat. Environ. Biophys. 41, $235(2002)$

5. S. Incerti, A. Ivanchenko, M. Karamitros, A. Mantero, P.Moretto, H.N. Tran, B. Mascialino, C. Champion, V.N. Ivanchenko, M.A. Bernal, Z. Fracis, C. Villagrasa, G. Baldacchino, P. Guèye, R. Capra, P. Nieminen, C. Zacharatou, Med. Phys. 37, 4692 (2010)

6. H. Nikjoo, D.T. Goodhead, Phys. Med. Biol. 36, 229 (1991) 
7. R.W. Schulte, A.J. Wroe, V.A. Bashkirov, G.Y. Garty, A. Breskin, R. Chechik, S. Shchemelinin, E. Gargioni, B. Grosswendt, A.B. Rosenfeld, Z. Med. Phys. 18, 286 (2008)

8. S. Pszona, J. Kula, S. Marjanska, Nucl. Instrum. Meth. Phys. Res. A 447, 601 (2000)

9. G. Garty, S. Shchemelinin, A. Breskin, R. Chechik, I. Orion, G.P. Guedes, R. Schulte, V. Bashkirov, B. Grosswendt, Radiat. Prot. Dosim. 99, 325 (2002)

10. G. Garty, S. Shchemelinin, A. Breskin, R. Chechik, G. Assaf, I. Orion, V. Bashkirov, R. Schulte, B. Grosswendt, Nucl. Instrum. Meth. Phys. Res. A 492, 212 (2002)

11. S. Shchemelinin, S. Pszona, G. Garty, A. Breskin, R. Chechik, NIM A 438, 447 (1999)

12. M.U. Bug, G. Hilgers, H. Nettelbeck, H. Rabus, PTBMitteilungen 121, 127 (2011)

13. V.T. Jordanov, G.F. Knoll, A.C. Huber, J.A. Pantazis, NIM A 353, 261 (1994)

14. J.F. Ziegler, M.D. Ziegler, J.P. Biersack, SRIM - The Stopping and Range of Ions in Matter Version 2006.02 (2006), http://www.srim.org/
15. ISO/IEC Guide 98-3:2008, Uncertainty of measurement Part 3: Guide to the expression of uncertainty in measurement (GUM:1995), http://www.bipm.org/utils/ common/documents/jcgm/JCGM_100_\discretionary2008_E.pdf

16. R. Böttger, Energy calibration of the PTB-VdG accelerator, PTB-report, PTB-6.41-02-2 (2002)

17. H. Bethe, Ann. Phys. 397, 325 (1930)

18. B. Grosswendt, Radiat. Prot. Dosim. 122, 404 (2006)

19. G. Hilgers, Rad. Meas. 45, 1228 (2010)

20. E. Gargioni, B. Grosswendt, NIM A 580, 81 (2007)

21. M.U. Bug, E. Gargioni, H. Nettelbeck, W.Y. Baek, G. Hilgers, A.B. Rosenfeld, H. Rabus, Phys. Rev. E 88, $043308(2013)$

Open Access This is an open access article distributed under the terms of the Creative Commons Attribution License (http://creativecommons.org/licenses/by/4.0), which permits unrestricted use, distribution, and reproduction in any medium, provided the original work is properly cited. 